\title{
Parisian types of ruin probabilities for a class of dependent risk-reserve processes
}

\author{
Bladt, Mogens; Nielsen, Bo Friis; Peralta, Oscar
}

Published in:

Scandinavian Actuarial Journal

Link to article, DOI:

10.1080/03461238.2018.1483420

Publication date:

2018

Document Version

Peer reviewed version

Link back to DTU Orbit

Citation (APA):

Bladt, M., Nielsen, B. F., \& Peralta, O. (2018). Parisian types of ruin probabilities for a class of dependent riskreserve processes. Scandinavian Actuarial Journal, 2019(1), 32-61.

https://doi.org/10.1080/03461238.2018.1483420

\section{General rights}

Copyright and moral rights for the publications made accessible in the public portal are retained by the authors and/or other copyright owners and it is a condition of accessing publications that users recognise and abide by the legal requirements associated with these rights.

- Users may download and print one copy of any publication from the public portal for the purpose of private study or research.

- You may not further distribute the material or use it for any profit-making activity or commercial gain

- You may freely distribute the URL identifying the publication in the public portal

If you believe that this document breaches copyright please contact us providing details, and we will remove access to the work immediately and investigate your claim. 


\section{Parisian types of ruin probabilities for a class of dependent risk-reserve processes}

\section{Mogens Bladt, Bo Friis Nielsen \& Oscar Peralta}

To cite this article: Mogens Bladt, Bo Friis Nielsen \& Oscar Peralta (2018): Parisian types of ruin probabilities for a class of dependent risk-reserve processes, Scandinavian Actuarial Journal, DOI: 10.1080/03461238.2018.1483420

To link to this article: https://doi.org/10.1080/03461238.2018.1483420

\section{Published online: 29 Jun 2018.}

\section{Submit your article to this journal $\widetilde{ }$}

山 Article views: 26

View Crossmark data ¿ 


\title{
Parisian types of ruin probabilities for a class of dependent risk-reserve processes
}

\author{
Mogens Bladt ${ }^{a}$, Bo Friis Nielsen ${ }^{b}$ and Oscar Peralta ${ }^{b}$ \\ ${ }^{a}$ Department of Mathematical Sciences, University of Copenhagen, Copenhagen, Denmark; ${ }^{\mathrm{b}}$ DTU COMPUTE, \\ Department of Applied Mathematics and Computer Science, Technical University of Denmark, Lyngby, Denmark
}

\begin{abstract}
For a rather general class of risk-reserve processes, we provide an exact method for calculating different kinds of ruin probabilities, with particular emphasis on variations over Parisian type of ruin. The risk-reserve processes under consideration have, in general, dependent phase-type distributed claim sizes and inter-arrivals times, whereas the movement between claims can either be linear or follow a Brownian motion with linear drift. For such processes, we provide explicit formulae for classical, Parisian and cumulative Parisian types of ruin (for both finite and infinite time horizons) when the clocks are phase-type distributed. An erlangization scheme provides an efficient algorithmic methods for calculating the aforementioned ruin probabilities with deterministic clocks. Special attention is drawn to the construction of specific dependency structures, and we provide a number of numerical examples to study its effect on probabilities.
\end{abstract}

\section{ARTICLE HISTORY}

Received 3 April 2018

Accepted 27 May 2018

\section{KEYWORDS}

Sparre-Andersen; Brownian motion; ruin probability; (cumulative) Parisian ruin; fluid flow; order statistics; dependency; Baker copula; phase-type distributions; erlangization; Lévy process

\section{JEL CLASSIFICATION} $\mathrm{G} 22$

\section{Summary}

Consider a risk-reserve process $\left\{R_{t}\right\}_{t \geq 0}$ on the form

$$
R_{t}=u+t+\sigma W_{t}-\sum_{i=1}^{N(t)} U_{i}
$$

where $R_{t}$ describes the reserve of an insurance company at time $t, u$ being the initial capital, $\left\{U_{i}\right\}_{i \geq 1}$ identically distributed claim sizes, $\{N(t)\}_{t \geq 0}$ the arrival process of the claims and $\left\{W_{t}\right\}_{t \geq 0}$ is an independent standard Brownian motion. Let $0=S_{0}<S_{1}<S_{2}<\cdots$ denote the arrival epochs of $\{N(t)\}_{t \geq 0}$ and $T_{i}=S_{i}-S_{i-1}, i \geq 1$, the corresponding inter-arrival times, which we assume are also identically distributed. The dependence structure between the different random variables will be defined later.

Assuming both claims and inter-arrivals to be phase-type distributed, we embed the risk-reserve process $\left\{R_{t}\right\}_{t \geq 0}$ into an equivalent fluid flow processes (with a possible Brownian component), which we call fluid flow risk process. This will allow us to introduce dependencies in a simple and controlled manner using the idea underlying the Baker copula by representing the phase-type distributions in terms of their order statistics. In this way, we shall be able to control the dependency in terms of, e.g. the Pearson correlation coefficient. The possibility of dependency between inter-arrival times and claims is also considered in the present setup.

CONTACT Mogens Bladt bladt@math.ku.dk Department of Mathematical Sciences, University of Copenhagen,

Universitetsparken 5, DK-2100 Copenhagen, Denmark 
Fluid flow processes, apart from being convenient in constructing the dependency structures, also allows for calculating ruin probabilities of different kinds like the usual infinite time-horizon, finite time-horizon, Parisian and finite time-horizon Parisian. Parisian ruin happens if a risk-reserve process, upon becoming negative, does not recover within some prescribed finite time. This time, which can also be random, is referred to as the clock. The clock, which can either be restarted every time the reserve becomes negative (classical Parisian ruin) or temporarily stopped during positive excursions (cumulative Parisian ruin), will be assumed to be i.i.d. phase-type distributed. An erlangization scheme (Section 5) will then enable us to effectively calculate the probability of Parisian ruin with deterministic clocks. Other types of Parisian ruin, like e.g. random clocks for the classical Parisian ruin sampled only once (see Loeffen et al. In Press), will not be considered in this paper.

\section{Background}

The idea of replacing risk-reserve processes by an equivalent fluid flow process goes back at least to Asmussen (1995). Formulae for the infinite time-horizon probability of ruin in the fluid flow setting are well known, which are usually referred to as first passage probabilities. We stress that studying risk processes through equivalent fluid flow processes has been used extensively; see Badescu \& Landriault (2009) for a survey. One can achieve a great level of generality with this approach. For instance, the model constructed in Ramaswami (2006) consists of a Markov-modulated risk process with statedependent claim sizes, with extra claims arising whenever the modulating process changes states. Such a process is governed by a Markovian Arrival Process with state- or jump-dependent claim sizes. The way this process is defined makes it attractive if one is looking to create dependence through Markov modulation. However, for the sake of generality, certain properties may become opaque and not easy to understand nor compute. One of them is the following simple question: how can one guarantee that the inter-arrival times and claim sizes follow given marginal distributions while keeping given dependence structures? In Section 6, we develop a systematic construction for different dependence scenarios while keeping the marginal distributions fixed. Our construction will be based on ideas underlying Baker's copula; see Baker (2008) and Lin \& Huang (2010).

The literature on risk models with fixed marginals and dependencies within its elements, or their queueing duals, goes back at least to Conolly (1968) for the case of the $M / M / 1$ queue with dependency between pairs of service times and inter-arrival times. While several papers are devoted to the $M / M / 1$ queue (see Borst et al. 1992 and references therein), this kind of dependency has been pursued for more general models in, e.g. Borst et al. (1992) for the $M / G / 1$ queue and (Müller 2000) for the $G / G / 1$ queue. In the risk modelling setting, dependency between inter-arrival times and claim sizes was introduced via threshold structures in Albrecher \& Boxma (2004) and Boudreault et al. (2006) for the Cramér-Lundberg process, later to be extended to the Sparre-Andersen process in Albrecher et al. (2014). In Badescu et al. (2009), a Sparre-Andersen process whose inter-arrival times and claim sizes follow an Assaf's bivariate phase-type distribution (Assaf et al. 1984) is studied by using a fluid flow process embedding. This approach is further extended in Badila et al. (2014) and Badila et al. (2015) by using the more general class of multivariate matrix-exponential distributions (Bladt \& Nielsen 2010), though their method is entirely analytical being based on excursion theory and the Wiener-Hopf factorization. Another relevant paper is Avram et al. (2016), where dependency between pairs arises by defining certain bivariate distribution with one phase-type-distributed marginal.

The concept of Parisian ruin with deterministic clocks was first studied in Dassios \& Wu (2008) for a Cramér-Lundberg process with exponential claim sizes. The results were later generalized to the case of spectrally negative Lévy processes in Czarna \& Palmowski (2011) and Loeffen et al. (2013) for the case of deterministic clocks, and in Landriault et al. (2014) and Baurdoux et al. (2016) for the case of random clocks, Erlang and exponential, respectively. In Czarna et al. (2017), the distribution of the number of claims leading to Parisian ruin is computed for the Cramér-Lundberg process. A related paper is Albrecher \& Ivanovs (2017), where a discretely observed ruin model can only be declared 
ruined if it is ever below 0 at its observation points driven by a Poisson process; this coincides with the case of Parisian ruin with exponentially distributed clocks.

\section{Phase-type distributions and fluid flow processes}

Let $\left\{X_{t}\right\}_{t \geq 0}$ denote a Markov jump process on a state-space $\{1,2, \ldots, p, p+1\}$, where states $1,2, \ldots, p$ are transient and state $p+1$ is absorbing. Then $\left\{X_{t}\right\}_{t \geq 0}$ has an intensity matrix on the form

$$
\Lambda=\left(\begin{array}{ll}
T & t \\
0 & 0
\end{array}\right)
$$

where $\boldsymbol{T}$ is a $p \times p$ sub-intensity matrix and $\boldsymbol{t}$ is a $p$-dimensional column vector. Since rows sum to zero, we have that $\boldsymbol{t}=-\boldsymbol{T} \boldsymbol{e}$, where $\boldsymbol{e}$ is the $p$-dimensional column vector of ones. Let $\pi_{i}=\mathbb{P}\left(X_{0}=i\right)$, $i=1, \ldots, p, \pi=\left(\pi_{1}, \ldots, \pi_{p}\right)$ and assume that $\mathbb{P}\left(X_{0}=p+1\right)=0$. Then we say that the time until absorption

$$
\tau=\inf \left\{t \geq 0 \mid X_{t}=p+1\right\}
$$

has a phase-type distribution with representation $(\pi, T)$ and we write $\tau \sim \operatorname{PH}(\pi, T)$ or $\tau \sim$ $\mathrm{PH}_{p}(\boldsymbol{\pi}, \boldsymbol{T})$. The set of transient states $\{1,2, \ldots, p\}$ is called the phase-space of the representation. Phase-type representations are by no means unique, a feature which will prove to be useful in the following sections when representing a phase-type distribution as a mixture of the distributions of its order statistics. For further notation and background on phase-type distribution, we refer to, e.g. Bladt (2005).

A fluid flow process with Brownian components initiated at level $u \in \mathbb{R}$ is a Markov additive process $\left\{\left(V_{t}, J_{t}\right)\right\}_{t \geq 0}$ (see Asmussen 2003, p. 309ff.) where $\left\{J_{t}\right\}_{t \geq 0}$ is a Markov jump process with finite state-space, $E$ say, and additive component $\left\{V_{t}\right\}_{t \geq 0}$ on the form

$$
V_{t}=u+\int_{0}^{t} r_{J_{s}} \mathrm{~d} s+\int_{0}^{t} \sigma_{J_{s}} \mathrm{~d} W_{s},
$$

where $\left\{W_{t}\right\}_{t \geq 0}$ is a standard Brownian motion independent of $\left\{J_{t}\right\}_{t \geq 0}$, and for every $i \in E, r_{i} \in \mathbb{R}$ and $\sigma_{i} \geq 0$. Define

$$
\begin{aligned}
E_{\sigma} & =\left\{i \in E: \sigma_{i}>0\right\}, \\
E_{0} & =\left\{i \in E: \sigma_{i}=0, r_{i}=0\right\}, \\
E_{+} & =\left\{i \in E: \sigma_{i}=0, r_{i}>0\right\}, \text { and } \\
E_{-} & =\left\{i \in E: \sigma_{i}=0, r_{i}<0\right\} .
\end{aligned}
$$

A classical fluid flow process is obtained for $E_{\sigma}=\emptyset$, whereas $E=E_{\sigma}$ corresponds to a Markovmodulated Brownian motion. From here on, we will always consider the case $E_{0}=\emptyset$.

Consider the risk-reserve process (1) with either $\sigma=0$ or $\sigma>0$; such a process is sketched in Figure 1 for the case $\sigma=0$. In order to construct its equivalent fluid flow process, which we call a fluid flow risk process, define a process $\left\{\left(V_{t}, J_{t}\right)\right\}_{t \geq 0}$ (as treated in Asmussen (1995) or Badescu et al. (2005)) where the claim sizes are no longer vertical downward jumps but linear movements with slope -1 . This way, the fluid flow risk process $\left\{V_{t}\right\}_{t \geq 0}$ will be at the same level as $\left\{R_{t}\right\}_{t \geq 0}$ just after the claim occurs (see Figure 2). To achieve this, define a Markov process $\left\{J_{t}\right\}$ on the state-space $E_{+} \cup E_{-}$if $\sigma=0\left(E_{\sigma} \cup E_{-}\right.$if $\left.\sigma>0\right)$, where $E_{+}\left(E_{\sigma}\right)$ denotes the phase-space of $\operatorname{PH}(\boldsymbol{\alpha}, S)$ and $E_{-}$ the phase-space of $\mathrm{PH}(\boldsymbol{\beta}, \boldsymbol{T})$, with intensity matrix

$$
\Lambda=\left(\begin{array}{cc}
S & D_{12} \\
D_{21} & T
\end{array}\right)
$$

for some matrices $\boldsymbol{D}_{12} \geq \mathbf{0}$ and $\boldsymbol{D}_{21} \geq \mathbf{0}$ such that $\boldsymbol{S} \boldsymbol{e}+\boldsymbol{D}_{12} \boldsymbol{e}=\mathbf{0}$ and $\boldsymbol{T e}+\boldsymbol{D}_{21} \boldsymbol{e}=\mathbf{0}$. If $\sigma=0$, $D_{12}=\boldsymbol{s} \boldsymbol{\beta}$ and $\boldsymbol{D}_{21}=\boldsymbol{t} \boldsymbol{\alpha}$ then inter-arrivals and claims are all independent, and the model will be 


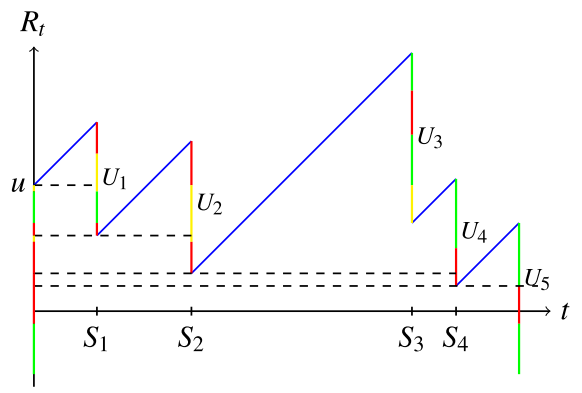

Figure 1. A risk-reserve process $\left\{R_{t}\right\}_{t \geq 0}$ without Brownian component $(\sigma=0)$. The jumps, caused by the phase-type distributed claims, are illustrated by vertical lines where the different colours refer to different states of the underlying Markov process. The ladder process on the vertical axis is obtained by projecting the underlying Markov processes of claims when reaching a local minimum.

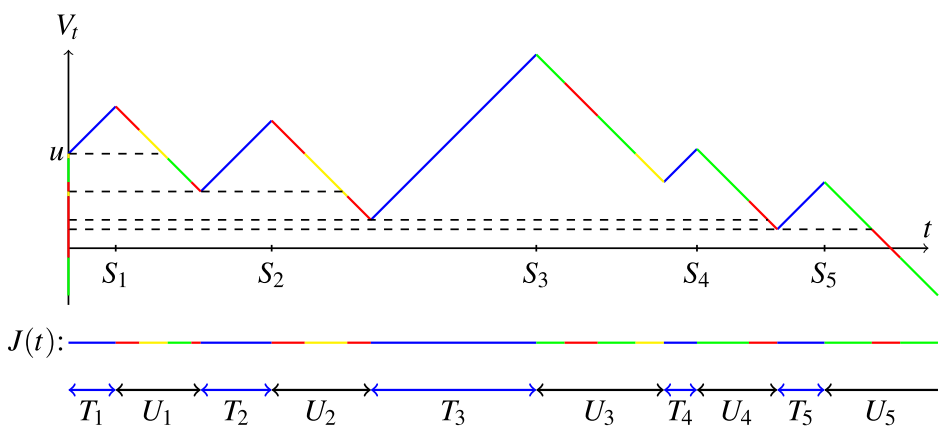

Figure 2. The risk-reserve process of Figure 1 converted into a fluid flow risk process $V_{t}$ by straightening out the vertical jumps into lines with slopes -1 . If the horizontal blue lines, representing the inter-arrival times $T_{i}, i=1,2, \ldots$, are phase-type distributed, the blue line below the coordinate system is generated by a terminating Markov jump process $\left\{J_{t}\right\}_{t \geq 0}$. The ladder process on the vertical axis is identical to the one obtained for the risk-reserve process in Figure 1.

equivalent to a Sparre-Andersen process with phase-type distributed inter-arrivals and claims. The matrices $\boldsymbol{D}_{12}$ and $\boldsymbol{D}_{21}$ define the dependency structure between inter-arrivals and claims. In order to gain flexibility in the choice of dependency, it might be necessary to increase the dimensions of the phase-type representations. This will also increase the dimensions of $\boldsymbol{D}_{12}$ and $\boldsymbol{D}_{21}$. A procedure for increasing the dimension without increasing the number of parameters is the topic of Section 6.

\section{Ruin probabilities}

We consider risk-reserve processes, represented by their equivalent fluid flow risk processes, where their trajectories between claims are either linear increments or Brownian motions with (positive) drift. We assume positive drift of the risk-reserve process, and therefore of the equivalent fluid flow risk process as well.

\subsection{Linear inter-claim increments}

Let $\left\{\left(V_{t}, J_{t}\right)\right\}_{t \geq 0}$ be a fluid flow risk process such that $E_{\sigma}=\emptyset, J_{0}=i \in E_{+}$and that $V_{0}=u>0$. Then $\left\{J_{t}\right\}_{t \geq 0}$ has state-space $E=E_{+} \cup E_{-}$, and we may assume w.l.o.g. that its intensity matrix can be written on the form

$$
\boldsymbol{\Lambda}=\left(\begin{array}{ll}
\boldsymbol{\Lambda}^{++} & \boldsymbol{\Lambda}^{+-} \\
\boldsymbol{\Lambda}^{-+} & \boldsymbol{\Lambda}^{--}
\end{array}\right)
$$


where, e.g. $\boldsymbol{\Lambda}^{++}$denotes the transition rates for transitions between states of $E_{+}$. If we have another look at Figures 1 and 2, we see that ruin happens if and only if the descending ladder process on the vertical axis ever reaches zero. We let $\boldsymbol{\beta}^{+-}$denote the $\left|E_{+}\right| \times\left|E_{-}\right|$-matrix whose $i j$-th entry is the probability that $R_{t}$ (or equivalently, $V_{t}$ ) downcrosses level $u$ in state $j \in E_{-}$given that $J_{0}=i \in E_{+}$. We let $\boldsymbol{D}$ (for "descending") denote the intensity matrix of the projected descending ladder process. Then it is straightforward (see, e.g. Asmussen (1995) and Bean et al. (2005)) that the probability of downcrossing 0 by $\left\{V_{t}\right\}_{t \geq 0}$ for the first time while $\left\{J_{t}\right\}_{t \geq 0}$ is in state $j \in E_{-}$is given by

$$
\boldsymbol{e}_{i}^{\prime} \boldsymbol{\beta}^{+-} e^{\boldsymbol{D} u} \boldsymbol{e}_{j}=\left(\boldsymbol{e}_{i}^{\prime} \boldsymbol{\beta}^{+-} e^{\boldsymbol{D} u}\right)_{j},
$$

where $\boldsymbol{e}_{i}$ denotes a column vector whose elements are all zero except its $i$-th one, which has the value 1. The matrices $\boldsymbol{\beta}^{+-}$and $\boldsymbol{D}$ can be calculated by the iteration scheme given in Bladt \& Nielsen (2017), Theorem 9.4.2.

Since infinite time-horizon ruin happens if and only if the descending ladder process ever reaches level 0 , we obtain the ruin probability by summing over $j \in E_{-}$in (4). This proves the following standard result (see, e.g. Asmussen 1995 or Bladt \& Nielsen 2017).

Theorem 4.1: The infinite time-horizon probability of ruin for the fluid flow risk process $\left\{\left(V_{t}, J_{t}\right)\right\}_{t \geq 0}$ (with $E_{\sigma}=\emptyset, V_{0}=u>0$ and $J_{0}=i \in E_{+}$) is given by

$$
\boldsymbol{e}_{i}^{\prime} \boldsymbol{\beta}^{+-} e^{\boldsymbol{D} u} \boldsymbol{e} .
$$

The matrices $\boldsymbol{\beta}^{+-}$and $\mathbf{D}$ can be calculated by an iteration scheme (see Asmussen 1995 or Bladt \& Nielsen 2017, Theorem 9.4.2.).

Remark 4.2: In the case of no Brownian component, $\boldsymbol{\beta}^{+-}$is the minimal non-negative solution of the nonsymmetric algebraic Riccati equation

$$
\boldsymbol{\Lambda}^{++} \boldsymbol{\beta}^{+-}+\boldsymbol{\beta}^{+-} \boldsymbol{\Lambda}^{--}+\boldsymbol{\beta}^{+-} \boldsymbol{\Lambda}^{-+} \boldsymbol{\beta}^{+-}+\boldsymbol{\Lambda}^{+-}=\mathbf{0},
$$

and $\boldsymbol{D}=\boldsymbol{\Lambda}^{--}+\boldsymbol{\Lambda}^{-+} \boldsymbol{\beta}^{+-}$, which may be solved in a number of ways (see Bean et al. 2005).

Define $\left\{d_{n}\right\}_{n \geq 1}$ and $\left\{u_{n}\right\}_{n \geq 1}$ as the (possibly finite) set of sequential points in time at which the process $\left\{V_{t}\right\}_{t \geq 0}$ downcrosses and upcrosses 0 , respectively. More precisely, we let $u_{0}=0$ and for $n \geq 1$

$$
\begin{aligned}
& d_{n}=\inf \left\{t \geq u_{n-1}: V_{t}<0\right\}, \\
& u_{n}=\inf \left\{t>d_{n}: V_{t} \geq 0\right\},
\end{aligned}
$$

so that $\left\{\left[u_{n-1}, d_{n}\right)\right\}_{n \geq 1}$ corresponds to the sequence of excursions of $\left\{V_{t}\right\}_{t \geq 0}$ above zero, and $\left\{\left[d_{n}, u_{n}\right)\right\}_{n \geq 1}$ corresponds to the sequence of excursions of $\left\{V_{t}\right\}_{t \geq 0}$ below zero. For all $n \geq 1$, let

$$
h_{n}=\int_{d_{n}}^{u_{n}} \mathbb{1}_{J_{s} \in E_{+}} \mathrm{d} s
$$

( $h_{n}=0$ for $d_{n}=+\infty$ ) denote the total amount of actual time, in the sense of the equivalent process $\left\{R_{t}\right\}_{t \geq 0}$, accumulated in the $n$-th sub-excursion below 0 .

Definition 4.3 (Parisian ruin): Let $L_{1}, L_{2}, \cdots \sim F$ be i.i.d. random variables, referred to as clocks. We say that $\left\{\left(V_{t}, J_{t}\right)\right\}_{t \geq 0}$ (with $E_{\sigma}=\emptyset, V_{0}=u>0$ and $J_{0}=i \in E_{+}$) gets ruined in the Parisian way with $F$-distributed clocks if there exists some $n \geq 1$ such that $L_{n}<h_{n}$. We let $\psi_{i}^{L}(u)$ denote the conditional probability Parisian ruin given that $J_{0}=i$. 
Definition 4.4 (Cumulative Parisian ruin): Cumulative Parisian ruin with an $F$-distributed clock happens if $L<\sum_{n=1}^{\infty} h_{n}$ for some independent non-negative random variable $L \sim F$, i.e. the process is allowed to be negative up to an accumulated total of $L$ units of time before declaring ruin. We let $\phi_{i}^{L}(u)$ denote the conditional probability of cumulative Parisian ruin given that $J_{0}=i$.

As for Parisian ruin, the concept of cumulative Parisian ruin was originally defined for Lévy processes in Guérin \& Renaud (2017).

In the Parisian setting, a clock $L_{n}$ is generated if and when $\left\{V_{t}\right\}_{t \geq 0}$ downcrosses 0 for the $n$-th time. If $\left\{V_{t}\right\}_{t \geq 0}$ can manage to get back into positive before $h_{n}$ exceeds $L_{n}$, then Parisian ruin has been avoided in this sub-excursion below 0 . The process $\left\{V_{t}\right\}_{t \geq 0}$ may thus get ruined in the usual sense, possibly become negative several times, but still survive in the Parisian setting.

Now consider the first downcrossing of 0 by $\left\{V_{t}\right\}_{t \geq 0}$. By the strong Markov property, it is enough to study the scenario in which $V_{0}=0$ and $J_{0}=i$ for $i \in E_{-}$. Under these assumptions, we define

$$
\tau=\inf \left\{t>0: V_{t}=0, J_{t} \notin E_{-}\right\} \text {and } h=\int_{0}^{\tau} \mathbb{1}_{V_{s}<0, J_{s} \notin E_{-}} \mathrm{d} s,
$$

so that $h$ is the accumulated time within the first sub-excursion below 0 . For $i \in E_{-}$, we let

$$
\bar{\psi}_{i j}(L)=\mathbb{P}\left(L>h, J_{\tau}=j \mid V_{0}=0, J_{0}=i\right)
$$

denote the probability that the first excursion below zero terminates prior to time $L \sim F$ by upcrossing in state $j \in E_{+}$. Let $\overline{\boldsymbol{\Psi}}(L)=\left\{\bar{\psi}_{i j}(L)\right\}_{i \in E_{-}, j \in E_{+}}$denote the corresponding matrix. Then have the following result.

Theorem 4.5: The conditional probability of Parisian ruin given that $J_{0}=i$, is given by

$$
\psi_{i}^{L}(u)=\boldsymbol{e}_{i}^{\prime} \boldsymbol{\beta}^{+-} e^{\boldsymbol{D} u}\left(\boldsymbol{I}-\overline{\boldsymbol{\Psi}}(L) \boldsymbol{\beta}^{+-}\right)^{-1}(\boldsymbol{e}-\overline{\boldsymbol{\Psi}}(L) \boldsymbol{e}),
$$

where I denotes an identity matrix of appropriate dimension.

Proof: The probability of Parisian ruin equals the probability that there is an excursion below zero which does not recover on time. Thus,

$$
\begin{aligned}
\psi_{i}^{L}(u) & =\sum_{n=1}^{\infty} \mathbb{P}\left(\bigcap_{j<n}\left\{L_{j}>h_{j}\right\}, L_{n}<h_{n} \mid V_{0}=u, J_{0}=i\right) \\
& =\sum_{n=1}^{\infty} \boldsymbol{e}_{i}^{\prime} \boldsymbol{\beta}^{+-} e^{\boldsymbol{D} u}\left(\overline{\boldsymbol{\Psi}}(L) \boldsymbol{\beta}^{+-}\right)^{n-1}(\boldsymbol{e}-\overline{\boldsymbol{\Psi}}(L) \boldsymbol{e}) \\
& =\boldsymbol{e}_{i}^{\prime} \boldsymbol{\beta}^{+-} e^{\boldsymbol{D} u}\left(\sum_{n=0}^{\infty}\left(\overline{\boldsymbol{\Psi}}(L) \boldsymbol{\beta}^{+-}\right)^{n}\right)(\boldsymbol{e}-\overline{\boldsymbol{\Psi}}(L) \boldsymbol{e}) \\
& =\boldsymbol{e}_{i}^{\prime} \boldsymbol{\beta}^{+-} e^{\boldsymbol{D} u}\left(\boldsymbol{I}-\overline{\boldsymbol{\Psi}}(L) \boldsymbol{\beta}^{+-}\right)^{-1}(\boldsymbol{e}-\overline{\boldsymbol{\Psi}}(L) \boldsymbol{e}) .
\end{aligned}
$$

Notice that the positive drift assumption of $\left\{V_{t}\right\}_{t \geq 0}$ implies that $\left(\boldsymbol{\beta}^{+-} \boldsymbol{e}\right)_{j}<1$ for $j \in E_{+}$. This in turn implies that $\overline{\boldsymbol{\Psi}}(L) \boldsymbol{\beta}^{+-}$is a sub-tranisiton matrix, so that $\boldsymbol{I}-\overline{\boldsymbol{\Psi}}(L) \boldsymbol{\beta}^{+-}$in (6) is indeed invertible.

From here on, let $\otimes$ and $\oplus$ denote the Kronecker product and Kronecker sum operator between matrices, respectively. For phase-distributed clocks, we obtain an explicit formula for the probability of Parisian ruin. 
Theorem 4.6: Let $L \sim P H_{\ell}(\kappa, K)$ and denote by $E_{\ell}$ its phase-space. Define a fluid flow risk process $\left\{\left(V_{t}^{L}, J_{t}^{L}\right)\right\}_{t \geq 0}$, where $\left\{J_{t}^{L}\right\}_{t \geq 0}$ has intensity matrix

$$
\left(\begin{array}{cc}
\Lambda^{++} \oplus K & \Lambda^{+-} \otimes I \\
\Lambda^{-+} \otimes I & \Lambda^{--} \otimes I
\end{array}\right)
$$

Let $\boldsymbol{\alpha}_{L}^{-+}$denote the matrix of upcrossing probabilities of such a fluid flow risk process, which can be calculated by the iteration scheme in Theorem 9.4.2 of Bladt \& Nielsen (2017). Then

$$
\overline{\boldsymbol{\Psi}}(L)=(\boldsymbol{I} \otimes \boldsymbol{\kappa}) \boldsymbol{\alpha}_{L}^{-+}(\boldsymbol{I} \otimes \boldsymbol{e}) .
$$

Remark 4.7: Again, $\boldsymbol{\alpha}_{L}^{-+}$may is the minimal non-negative solution of a nonsymmetric algebraic Riccati equation, namely

$$
\left(\Lambda^{--} \otimes I\right) \alpha_{L}^{-+}+\alpha_{L}^{-+}\left(\Lambda^{++} \oplus K\right)+\alpha_{L}^{-+}\left(\Lambda^{+-} \otimes I\right) \alpha_{L}^{-+}+\left(\Lambda^{-+} \otimes I\right)=\mathbf{0} .
$$

For the association of $\boldsymbol{\alpha}_{L}^{-+}$as the minimal solution to the stated Riccati equation and its computation, we refer to Bean et al. (2005).

Proof of Theorem 4.6.: Let $J_{0}^{L} \sim\left(\boldsymbol{e}_{i}^{\prime} \otimes \boldsymbol{\kappa}, \mathbf{0}\right)$ and write $J_{t}^{L}=\left(i_{t}, i_{t}^{L}\right)$. Then $i_{0}=i$ and $i_{0}^{L} \sim \boldsymbol{\kappa}$. According to (7), $\left\{i_{t}^{L}\right\}$ will remain fixed or frozen for all $t \geq 0$ such that $i_{t} \in E_{-}$. While $i_{t} \in E_{+}$, $\left\{i_{t}^{L}\right\}$ will develop according to the sub-intensity matrix $K$, so that $\left\{i_{t}^{L}\right\}$ is a terminating process. On the other hand, $\left\{i_{t}\right\}$ will simply develop according to the original matrix $\boldsymbol{\Lambda}$ up to the (possible) termination of $\left\{i_{t}^{L}\right\}$. With this in mind, let $\alpha_{L}^{-+}$be the $\left|E_{-} \times E_{\ell}\right| \times\left|E_{+} \times E_{\ell}\right|$-matrix of upcrossing probabilities for the fluid flow risk process $\left\{\left(V_{t}^{L}, J_{t}^{L}\right)\right\}_{t \geq 0}$. Let

$$
\tau_{L}=\inf \left\{t>0: V_{t}^{L}=0, J_{t}^{L} \notin E_{-} \times E_{\ell}\right\} .
$$

Then, for all $i \in E_{-}$and $j \in E_{+}$,

$$
\begin{aligned}
\bar{\psi}_{i j}(L) & =\sum_{k_{2}=1}^{\ell} \sum_{k_{1}=1}^{\ell} \mathbb{P}\left(\tau_{L}<\infty, i_{0}^{L}=k_{1}, i_{\tau_{L}}=j, i_{\tau_{L}}^{L}=k_{2} \mid V_{0}=0, i_{0}=i\right) \\
& =\sum_{k_{2}=1}^{\ell} \sum_{k_{1}=1}^{\ell} \kappa_{k_{1}}\left(\boldsymbol{\alpha}_{L}^{-+}\right)_{\left((i-1) \ell+k_{1},(j-1) \ell+k_{2}\right)} \\
& =\left((\boldsymbol{I} \otimes \boldsymbol{\kappa}) \boldsymbol{\alpha}_{L}^{-+}(\boldsymbol{I} \otimes \boldsymbol{e})\right)_{i j},
\end{aligned}
$$

so the result follows.

The case of cumulative Parisian ruin is similar.

Theorem 4.8: The conditional probability of cumulative Parisian ruin with clock $L \sim P H(\kappa, K)$ given that $J_{0}=i$ is given by

$$
\phi_{i}^{L}(u)=\left(\boldsymbol{e}_{i}^{\prime} \boldsymbol{\beta}^{+-} e^{D u} \otimes \boldsymbol{\kappa}\right)\left(\boldsymbol{I}-\boldsymbol{\alpha}_{L}^{-+}\left(\boldsymbol{\beta}^{+-} \otimes \boldsymbol{I}\right)\right)^{-1}\left(\boldsymbol{e}-\boldsymbol{\alpha}_{L}^{-+} \boldsymbol{e}\right),
$$

where $\boldsymbol{\alpha}_{L}^{-+}$is defined in Theorem 4.6.

Proof: The probability distribution of the phases at the first downcrossing of level 0 is given by $\boldsymbol{e}_{i}^{\prime} \boldsymbol{\beta}^{+-} e^{\boldsymbol{D} u}$. As in the proof of Theorem 4.6, the clock $L$ is then initiated and we consider the process $\left\{\left(V_{t}^{L}, J_{t}^{L}\right)\right\}$ with upcrossing probabilities $\boldsymbol{\alpha}_{L}^{-+}$. If the process gets back to level 0 we need to keep 
information on the phase of $L$ until the next downcrossing, which is done with the term $\boldsymbol{\beta}^{+-} \otimes \boldsymbol{I}$. We can have any finite number $n$ of excursions below and above level $0(n \geq 0)$. Cumulative parisian ruin happens if one of the sub-excursions below 0 does not upcross level 0 . Thus,

$$
\begin{aligned}
\phi_{i}^{L}(u) & =\sum_{n=1}^{\infty}\left(\boldsymbol{e}_{i}^{\prime} \boldsymbol{\beta}^{+-} e^{\boldsymbol{D} u} \otimes \boldsymbol{\kappa}\right)\left(\boldsymbol{\alpha}_{L}^{-+}\left(\boldsymbol{\beta}^{+-} \otimes \boldsymbol{I}\right)\right)^{n-1}\left(\boldsymbol{e}-\boldsymbol{\alpha}_{L}^{-+} \boldsymbol{e}\right) \\
& =\left(\boldsymbol{e}_{i}^{\prime} \boldsymbol{\beta}^{+-} e^{\boldsymbol{D} u} \otimes \boldsymbol{\kappa}\right)\left(\boldsymbol{I}-\boldsymbol{\alpha}_{L}^{-+}\left(\boldsymbol{\beta}^{+-} \otimes \boldsymbol{I}\right)\right)^{-1}\left(\boldsymbol{e}-\boldsymbol{\alpha}_{L}^{-+} \boldsymbol{e}\right) .
\end{aligned}
$$

\subsection{Inter-claim Brownian motions}

Let $\left\{\left(V_{t}, J_{t}\right)\right\}_{t \geq 0}$ be a fluid flow risk process such that $E_{+}=\emptyset, J_{0}=i \in E_{\sigma}$ and $V_{0}=u$. Then $\left\{J_{t}\right\}_{t \geq 0}$ has state-space $E=E_{\sigma} \cup E_{-}$and its intensity matrix may w.l.o.g. be written on the form

$$
\boldsymbol{\Lambda}=\left(\begin{array}{ll}
\boldsymbol{\Lambda}^{\sigma \sigma} & \boldsymbol{\Lambda}^{\sigma-} \\
\Lambda^{-\sigma} & \Lambda^{--}
\end{array}\right)
$$

According to Asmussen (1995), the probability of $\left\{V_{t}\right\}_{t \geq 0}$ downcrossing 0 for the first time while $\left\{J_{t}\right\}_{t \geq 0}$ is in state $j \in E_{\sigma} \cup E_{-}$is given by

$$
\left(\boldsymbol{e}_{i}^{\prime} \boldsymbol{\beta}^{+-} e^{\boldsymbol{D} u}\right)_{j},
$$

where

$$
\boldsymbol{\beta}^{+-}=\left(\begin{array}{ll}
\boldsymbol{I} & \mathbf{0}
\end{array}\right), \quad \boldsymbol{D}=\left(\begin{array}{ll}
\boldsymbol{D}^{\sigma \sigma} & \boldsymbol{D}^{\sigma-} \\
\boldsymbol{D}^{-\sigma} & \boldsymbol{D}^{--}
\end{array}\right), \quad \boldsymbol{D}^{-\sigma}=\boldsymbol{\Lambda}^{-\sigma}, \quad \boldsymbol{D}^{--}=\boldsymbol{\Lambda}^{--},
$$

and $\left(\begin{array}{cc}D^{\sigma \sigma} & D^{\sigma-}\end{array}\right)$ is the non-negative solution to the fixed-point equation

$$
\left(\begin{array}{ll}
\boldsymbol{D}^{\sigma \sigma} & \boldsymbol{D}^{\sigma-}
\end{array}\right)=\frac{2}{\sigma^{2}}\left(\begin{array}{ll}
\mu \boldsymbol{I}+\boldsymbol{\Lambda}^{\sigma \sigma} & \boldsymbol{\Lambda}^{\sigma-}
\end{array}\right)\left(\eta \boldsymbol{I}-\left(\begin{array}{ll}
\boldsymbol{D}^{\sigma \sigma} & \boldsymbol{D}^{\sigma-} \\
\boldsymbol{D}^{-\sigma} & \boldsymbol{D}^{--}
\end{array}\right)\right)^{-1}-\left(\begin{array}{ll}
\omega \boldsymbol{I} & \mathbf{0}
\end{array}\right),
$$

with

$$
\mu=\eta+\frac{\sigma^{2} \eta^{2}}{2}, \quad \omega=\frac{1}{\sigma^{2}}+\sqrt{\frac{1}{\sigma^{4}}+\frac{2 \mu}{\sigma^{2}}},
$$

and $\eta>0$ chosen such that $\mu \geq-\boldsymbol{\Lambda}_{i i}$ for all $i$. Iterative algorithms to compute $\boldsymbol{\beta}^{+-}$and $\boldsymbol{D}$ can be found in Asmussen (1995) or in Section 9.6.2 of Bladt \& Nielsen (2017). Thus we have the following.

Theorem 4.9: The infinite time-horizon probability of ruin for the fluid flow risk process $\left\{\left(V_{t}, J_{t}\right)\right\}_{t \geq 0}$ (with $E_{+}=\emptyset, V_{0}=u>0$ and $J_{0}=i \in E_{\sigma}$ ) is given by

$$
\boldsymbol{e}_{i}^{\prime} \boldsymbol{\beta}^{+-} e^{D u} \boldsymbol{e} .
$$

For the case of Parisian ruin with a Brownian component, we need to take a slightly different approach to the one described in Section 4.1. If $E_{\sigma} \neq \emptyset$, then the process can become negative also at times between claims as a result of the Brownian motion. Also, from the law of the iterated logarithm for Brownian motions, it follows that if the fluid flow risk process is zero at time $t$, say, then any finite interval $[t, t+\delta), \delta>0$, contains an infinite number of points where the process is zero again. To avoid this complication, we employ the following standard trick (see, e.g. Loeffen et al. 2013 or Baurdoux et al. 2016). 
Fix $\epsilon>0$, let $u_{0}^{\epsilon}=0$, and for $n \geq 1$ define

$$
\begin{aligned}
& d_{n}^{\epsilon}=\inf \left\{t \geq u_{n-1}^{\epsilon}: V_{t}<-\epsilon\right\} \\
& u_{n}^{\epsilon}=\inf \left\{t>d_{n}^{\epsilon}: V_{t} \geq 0\right\},
\end{aligned}
$$

so that $\left\{d_{n}^{\epsilon}\right\}_{n \geq 1}$ and $\left\{u_{n}^{\epsilon}\right\}_{n \geq 1}$ corresponds to the alternating points in time in which $\left\{V_{t}\right\}_{t \geq 0}$ downcrosses level $-\epsilon$, later to upcross level 0 , respectively. For all $n \geq 1$, let

$$
h_{n}^{\epsilon}=\int_{d_{n}^{\epsilon}}^{u_{n}^{\epsilon}} \mathbb{1}_{J_{s} \in E_{\sigma}} \mathrm{d} s
$$

with the convention that $h_{n}^{\epsilon}=0$ for $d_{n}^{\epsilon}=\infty$. Then $h_{n}^{\epsilon}$ corresponds to total amount of actual time, in the sense of the equivalent process $\left\{R_{t}\right\}_{t \geq 0}$, accumulated in the interval $\left[d_{n}^{\epsilon}, u_{n}^{\epsilon}\right)$.

Definition 4.10 (Parisian and $\epsilon$-Parisian ruin): Let $L_{1}, L_{2}, \cdots \sim F$ be i.i.d. random variables, referred to as clocks. Then, with $E_{+}=\emptyset, V_{0}=u>0$ and $J_{0}=i \in E_{\sigma}$, we say that $\left\{\left(V_{t}, J_{t}\right)\right\}_{t \geq 0}$ gets ruined in the $\epsilon$-Parisian way with $F$-distributed clocks if there exists some $n \geq 1$ such that $L_{n}<h_{n}^{\epsilon}$. We denote by $\psi_{i}^{L}(u, \epsilon)$ the conditional probability of $\epsilon$-Parisian ruin given that $J_{0}=i$. The conditional probability of Parisian ruin given that $J_{0}=i$ is then defined as $\psi_{i}^{L}(u)=\lim _{\epsilon \downarrow} \psi_{i}^{L}(u, \epsilon)$.

Remark 4.11: The number of downcrossings of level zero is countable and for each downcrossing we may assign an independent clock. Thus, Parisian ruin could also be defined more directly avoiding the $\epsilon$-approximation, see p. 6 of Baurdoux et al. (2016). The numbering of the downcrossings, however, is not obvious, and we believe that Definition 4.10 will provide a more useful approach being directly linked to sample path properties.

At each point $d_{n}^{\epsilon}$, we thus associate a clock $L_{n}$, and if $\left\{V_{t}\right\}_{t \geq 0}$ can manage to become positive again before the clock rings (i.e. $L_{n}>h_{n}^{\epsilon}$ ), then $\epsilon$-Parisian ruin has been avoided in the interval $\left[d_{n}^{\epsilon}, u_{n}^{\epsilon}\right.$ ).

Definition 4.12 (Cumulative Parisian and $\epsilon$-cumulative Parisian ruin): Let $L \sim F$ be a clock. Then, with $E_{+}=\emptyset, V_{0}=u>0$ and $J_{0}=i \in E_{\sigma}$, we say that $\left\{\left(V_{t}, J_{t}\right)\right\}_{t \geq 0}$ gets ruined in the $\epsilon$-cumulative Parisian way with $F$-distributed clock $L$ if $L<\sum_{n=1}^{\infty} h_{n}^{\epsilon}$. We denote by $\phi_{i}^{L}(u, \epsilon)$ the conditional probability of $\epsilon$-cumulative Parisian ruin given that $J_{0}=i$. The conditional probability of cumulative Parisian ruin given that $J_{0}=i$ is then defined as $\phi_{i}^{L}(u)=\lim _{\epsilon \downarrow} \phi_{i}^{L}(u, \epsilon)$.

Now, let $V_{0}=-\epsilon, J_{0}=i$ with $i \in E_{\sigma} \cup E_{-}$, let $h$ be as in (5) and let $\tau_{L}$ be as in (9). Define the probabilities of $\epsilon$-recovery before $L$ by

$$
\bar{\psi}_{i j}(L, \epsilon)=\mathbb{P}\left(h<L, J_{\tau_{L}}=j \mid V_{0}=-\epsilon, J_{0}=i\right) \quad \text { for } \quad i \in E_{\sigma} \cup E_{-}, j \in E_{\sigma},
$$

and let $\overline{\boldsymbol{\Psi}}(L, \epsilon)=\left\{\bar{\psi}_{i j}(L, \epsilon)\right\}_{i \in E_{\sigma} \cup E_{-, j} \in E_{\sigma}}$.

Theorem 4.13: The probability of $\epsilon$-Parisian ruin with clocks of the L-type is given by

$$
\psi_{i}^{L}(u, \epsilon)=\boldsymbol{e}_{i}^{\prime} \boldsymbol{\beta}^{+-} e^{\boldsymbol{D}(u+\epsilon)}\left(\boldsymbol{I}-\overline{\boldsymbol{\Psi}}(L, \epsilon) \boldsymbol{\beta}^{+-} e^{y} \boldsymbol{D} \epsilon\right)^{-1}(\boldsymbol{e}-\overline{\boldsymbol{\Psi}}(L, \epsilon) \boldsymbol{e})
$$




\section{Proof:}

$$
\begin{aligned}
\psi_{i}^{L}(u) & =\sum_{n=1}^{\infty} \mathbb{P}\left(\cap_{j<n}\left\{L_{j}>h_{j}^{\epsilon}\right\}, L_{n}<h_{n}^{\epsilon} \mid V_{0}=u, J_{0}=i\right) \\
& =\sum_{n=1}^{\infty} \boldsymbol{e}_{i}^{\prime} \boldsymbol{\beta}^{+-} e^{y} \boldsymbol{D}(u+\epsilon)\left(\overline{\boldsymbol{\Psi}}(L, \epsilon) \boldsymbol{\beta}^{+-} e^{\boldsymbol{D} \epsilon}\right)^{n-1}(\boldsymbol{e}-\overline{\boldsymbol{\Psi}}(L, \epsilon) \boldsymbol{e}) \\
& =\boldsymbol{e}_{i}^{\prime} \boldsymbol{\beta}^{+-} e^{\boldsymbol{D}(u+\epsilon)}\left(\boldsymbol{I}-\overline{\boldsymbol{\Psi}}(L, \epsilon) \boldsymbol{\beta}^{+-} e^{\boldsymbol{D} \epsilon}\right)^{-1}(\boldsymbol{e}-\overline{\boldsymbol{\Psi}}(L, \epsilon) \boldsymbol{e}) .
\end{aligned}
$$

As in the proof of Theorem 4.5, one can argue that $V_{t} \rightarrow \infty$ as $t \rightarrow \infty$ implies that

$$
\left(\boldsymbol{\beta}^{+-} e^{\boldsymbol{D} \epsilon} \boldsymbol{e}\right)_{j}<1 \quad \text { for all } j \in E_{\sigma} \text { and } \epsilon>0,
$$

so that $\boldsymbol{I}-\overline{\boldsymbol{\Psi}}(L, \epsilon) \boldsymbol{\beta}^{+-} e^{\boldsymbol{D} \epsilon}$ in (10) is indeed invertible.

Just as in Section 4.1, $\bar{\Psi}(L, \epsilon)$ will be explicitly computable given the assumption that $L$ is phasetype distributed.

Theorem 4.14: If $L \sim P H_{\ell}(\boldsymbol{\kappa}, \boldsymbol{K})$, then

$$
\overline{\boldsymbol{\Psi}}(L, \epsilon)=(\boldsymbol{I} \otimes \boldsymbol{\kappa}) \boldsymbol{\alpha}_{L} e^{\boldsymbol{U}_{L} \epsilon}(\boldsymbol{I} \otimes \boldsymbol{e})
$$

where

$$
\boldsymbol{\alpha}_{L}=\left(\begin{array}{c}
\boldsymbol{I} \\
\boldsymbol{\alpha}_{L}^{-+}
\end{array}\right), \quad \boldsymbol{U}_{L}=\boldsymbol{U}_{L}^{\sigma \sigma},
$$

with $\boldsymbol{\alpha}_{L}^{-+}$and $\boldsymbol{U}_{L}^{\sigma \sigma}$ satisfying simultaneously the equations

$$
\begin{aligned}
& \boldsymbol{\alpha}_{L}^{-+}=\left(\eta \boldsymbol{\alpha}_{L}^{-+}+\boldsymbol{\Lambda}^{-\sigma} \otimes \boldsymbol{I}+\left(\boldsymbol{\Lambda}^{--} \otimes \boldsymbol{I}\right) \boldsymbol{\alpha}_{L}^{-+}\right)\left(\eta \boldsymbol{I}-\boldsymbol{U}_{L}^{\sigma \sigma}\right)^{-1} \\
& \boldsymbol{U}_{L}^{\sigma \sigma}=\frac{2}{\sigma^{2}}\left(\mu \boldsymbol{I}+\boldsymbol{\Lambda}^{\sigma \sigma} \oplus \boldsymbol{K}+\left(\boldsymbol{\Lambda}^{\sigma-} \otimes \boldsymbol{I}\right) \boldsymbol{\alpha}_{L}^{-+}\right)\left(\eta \boldsymbol{I}-\boldsymbol{U}_{L}^{\sigma \sigma}\right)^{-1}-\omega \boldsymbol{I},
\end{aligned}
$$

with

$$
\mu=-\eta+\frac{\sigma^{2} \eta^{2}}{2}, \quad \omega=-\frac{1}{\sigma^{2}}+\sqrt{\frac{1}{\sigma^{4}}+\frac{2 \mu}{\sigma^{2}}},
$$

and $\eta>0$ chosen such that $\mu \geq-\boldsymbol{\Lambda}_{i i}$ for all $i$. Such solutions can be computed via iterative algorithms as the ones found in Asmussen (1995) or in Section 9.6.2 of Bladt \& Nielsen (2017).

Proof: Follows along the same lines as the proof of Theorem 4.6.

An argument analogous to the proof of Theorem 4.8 leads to the following.

Theorem 4.15: The probability of $\epsilon$-cumulative Parisian ruin with clock $L \sim P H(\kappa, \boldsymbol{K})$ conditional on $J_{0}=i$ is given by

$$
\phi_{i}^{L}(u, \epsilon)=\left(\boldsymbol{e}_{i}^{\prime} \boldsymbol{\beta}^{+-} e^{\boldsymbol{D}(u+\epsilon)} \otimes \boldsymbol{\kappa}\right)\left(\boldsymbol{I}-\boldsymbol{\alpha}_{L} e^{\boldsymbol{U}_{L} \epsilon}\left[\left(\boldsymbol{\beta}^{+-} e^{\boldsymbol{D} \epsilon}\right) \otimes \boldsymbol{I}\right]\right)^{-1}\left(\boldsymbol{e}-\boldsymbol{\alpha}_{L}^{-+} e^{\boldsymbol{U}_{L}} \boldsymbol{e}\right)
$$

As $\epsilon \downarrow 0$ we get the following results. 
Theorem 4.16: (1) The probability of Parisian ruin with clock $L \sim P H(\kappa, K)$ in the presence of Brownian component is given by

$$
\psi_{i}^{L}(u)=\boldsymbol{e}_{i}^{\prime} \boldsymbol{\beta}^{+-} e^{\boldsymbol{D} u}\left(\begin{array}{cc}
-\boldsymbol{D}^{\sigma \sigma}-(\boldsymbol{I} \otimes \boldsymbol{\kappa}) \boldsymbol{U}_{L}(\boldsymbol{I} \otimes \boldsymbol{e}) & -\boldsymbol{D}^{\sigma-} \\
-(\boldsymbol{I} \otimes \boldsymbol{\kappa}) \boldsymbol{\alpha}_{L}^{-+}(\boldsymbol{I} \otimes \boldsymbol{e}) & \boldsymbol{I}
\end{array}\right)^{-1}\left(\begin{array}{c}
-(\boldsymbol{I} \otimes \boldsymbol{\kappa}) \boldsymbol{U}_{L} \boldsymbol{e} \\
\boldsymbol{e}-(\boldsymbol{I} \otimes \boldsymbol{\kappa}) \boldsymbol{\alpha}_{L}^{-+} \boldsymbol{e}
\end{array}\right)
$$

(2) The probability of cumulative Parisian ruin with clock $L \sim P H(\kappa, K)$ in the presence of Brownian component is given by

$$
\phi_{i}^{L}(u)=\left(\boldsymbol{e}_{i}^{\prime} \boldsymbol{\beta}^{+-} e^{\boldsymbol{D} u} \otimes \kappa\right)\left(\begin{array}{cc}
-\boldsymbol{U}_{L}-\boldsymbol{D}^{\sigma \sigma} \otimes \boldsymbol{I} & -\boldsymbol{D}^{\sigma-} \otimes \boldsymbol{I} \\
-\boldsymbol{\alpha}_{L}^{-+} & \boldsymbol{I}
\end{array}\right)^{-1}\left(\begin{array}{c}
-U_{L} \boldsymbol{e} \\
\boldsymbol{e}-\boldsymbol{\alpha}_{L}^{-+} \boldsymbol{e}
\end{array}\right) .
$$

Proof: We first prove (14). Notice that

$$
\begin{aligned}
& \phi_{i}^{L}(u)=\lim _{\epsilon \rightarrow 0}\left(\boldsymbol{e}_{i}^{\prime} \boldsymbol{\beta}^{+-} e^{\boldsymbol{D}(u+\epsilon)} \otimes \boldsymbol{\kappa}\right)\left(\boldsymbol{I}-\left(\begin{array}{c}
\boldsymbol{I} \\
\boldsymbol{\alpha}_{L}^{-+}
\end{array}\right) e^{\boldsymbol{U}_{L} \epsilon}\left[\left(\boldsymbol{\beta}^{+-} e^{\boldsymbol{D} \epsilon}\right) \otimes \boldsymbol{I}\right]\right)^{-1} \\
& \times\left(\boldsymbol{e}-\left(\begin{array}{c}
\boldsymbol{I} \\
\boldsymbol{\alpha}_{L}^{-+}
\end{array}\right) e^{\boldsymbol{U}_{L} \epsilon} \boldsymbol{e}\right) \\
&=\lim _{\epsilon \rightarrow 0}\left(\boldsymbol{e}_{i}^{\prime} \boldsymbol{\beta}^{+-} e^{\boldsymbol{D}(u+\epsilon)} \otimes \boldsymbol{\kappa}\right)\left(\left(\begin{array}{cc}
\epsilon^{-1} \boldsymbol{I} & \mathbf{0} \\
\mathbf{0} & \boldsymbol{I}
\end{array}\right)\left[\boldsymbol{I}-\left(\begin{array}{c}
\boldsymbol{I} \\
\boldsymbol{\alpha}_{L}^{-+}
\end{array}\right) e^{\boldsymbol{U}_{L} \epsilon}\left[\left(\boldsymbol{\beta}^{+-} e^{D \epsilon}\right) \otimes \boldsymbol{I}\right]\right]\right)^{-1} \\
& \times\left(\begin{array}{cc}
\epsilon^{-1} \boldsymbol{I} & \mathbf{0} \\
\mathbf{0} & \boldsymbol{I}
\end{array}\right)\left(\boldsymbol{e}-\left(\begin{array}{c}
\boldsymbol{I} \\
\boldsymbol{\alpha}_{L}^{-+}
\end{array}\right) e^{\boldsymbol{U}_{L} \epsilon} \boldsymbol{e}\right) .
\end{aligned}
$$

Since

$$
\begin{aligned}
\lim _{\epsilon \rightarrow 0}\left(\begin{array}{cc}
\epsilon^{-1} \boldsymbol{I} & \mathbf{0} \\
\mathbf{0} & \boldsymbol{I}
\end{array}\right)\left(\boldsymbol{e}-\left(\begin{array}{c}
\boldsymbol{I} \\
\boldsymbol{\alpha}_{L}^{-+}
\end{array}\right) \boldsymbol{e}^{\boldsymbol{U}_{L} \epsilon} \boldsymbol{e}\right) \\
\quad=\lim _{\epsilon \rightarrow 0}\left(\begin{array}{c}
\epsilon^{-1}\left(\boldsymbol{e}-e^{\boldsymbol{U}_{L} \epsilon} \boldsymbol{e}\right) \\
\boldsymbol{e}-\boldsymbol{\alpha}_{L}^{-+} e^{\boldsymbol{U}_{L} \epsilon} \boldsymbol{e}
\end{array}\right) \\
\quad=\lim _{\epsilon \rightarrow 0}\left(\begin{array}{c}
\epsilon^{-1}\left(\boldsymbol{e}-\left(\boldsymbol{I}+\boldsymbol{U}_{L} \epsilon+o(\epsilon)\right) \boldsymbol{e}\right) \\
\boldsymbol{e}-\boldsymbol{\alpha}_{L}^{-+} e^{\boldsymbol{U}_{L} \epsilon} \boldsymbol{e}
\end{array}\right)=\left(\begin{array}{c}
-\boldsymbol{U}_{L} \boldsymbol{e} \\
\boldsymbol{e}-\boldsymbol{\alpha}_{L}^{-+} \boldsymbol{e}
\end{array}\right)
\end{aligned}
$$

and

$$
\begin{aligned}
\lim _{\epsilon \rightarrow 0}\left(\begin{array}{cc}
\epsilon^{-1} \boldsymbol{I} & \mathbf{0} \\
\mathbf{0} & \boldsymbol{I}
\end{array}\right)\left[\boldsymbol{I}-\left(\begin{array}{c}
\boldsymbol{I} \\
\boldsymbol{\alpha}_{L}^{-+}
\end{array}\right) e^{\boldsymbol{U}_{L} \epsilon}\left[\left(\boldsymbol{\beta}^{+-} e^{D \epsilon}\right) \otimes \boldsymbol{I}\right]\right] \\
\left.=\lim _{\epsilon \rightarrow 0}\left(\begin{array}{cc}
\epsilon^{-1} \boldsymbol{I} & \mathbf{0} \\
\mathbf{0} & \boldsymbol{I}
\end{array}\right)-\left(\begin{array}{c}
\epsilon^{-1} e^{\boldsymbol{U}_{L} \epsilon} \\
\boldsymbol{\alpha}_{L}^{-+} e^{\boldsymbol{U}_{L} \epsilon}
\end{array}\right)\left[\left(\begin{array}{ll}
\boldsymbol{I} & \mathbf{0}
\end{array}\right) e^{\boldsymbol{D} \epsilon}\right) \otimes \boldsymbol{I}\right] \\
=-\left(\begin{array}{cc}
\boldsymbol{U}_{L}+\boldsymbol{D}^{\sigma \sigma} \otimes \boldsymbol{I} & \boldsymbol{D}^{\sigma-} \otimes \boldsymbol{I} \\
\boldsymbol{\alpha}_{L}^{-+} & -\boldsymbol{I}
\end{array}\right),
\end{aligned}
$$


the result (14) follows. The nonsingularity of (15) follows by noticing that the matrix is the negative of a sub-intensity matrix. To prove (13),

$$
\begin{aligned}
\psi_{i}^{L}(u)= & \lim _{\epsilon \rightarrow 0} \boldsymbol{e}_{i}^{\prime} \boldsymbol{\beta}^{+-} e^{\boldsymbol{D}(u+\epsilon)}\left(\boldsymbol{I}-(\boldsymbol{I} \otimes \boldsymbol{\kappa})\left(\begin{array}{c}
\boldsymbol{I} \\
\boldsymbol{\alpha}_{L}^{-+}
\end{array}\right) e^{\boldsymbol{U}_{L} \epsilon}(\boldsymbol{I} \otimes \boldsymbol{e}) \boldsymbol{\beta}^{+-} e^{D \epsilon}\right)^{-1} \\
& \times\left(\boldsymbol{e}-(\boldsymbol{I} \otimes \boldsymbol{\kappa})\left(\begin{array}{c}
\boldsymbol{I} \\
\boldsymbol{\alpha}_{L}^{-+}
\end{array}\right) e^{\boldsymbol{U}_{L} \epsilon} \boldsymbol{e}\right) \\
= & \lim _{\epsilon \rightarrow 0} \boldsymbol{e}_{\boldsymbol{i}}^{\prime} \boldsymbol{\beta}^{+-} e^{\boldsymbol{D}(u+\epsilon)} \\
& \times\left(\left(\begin{array}{cc}
\epsilon^{-1} \boldsymbol{I} & \mathbf{0} \\
\mathbf{0} & \boldsymbol{I}
\end{array}\right)\left[\boldsymbol{I}-(\boldsymbol{I} \otimes \boldsymbol{\kappa})\left(\begin{array}{c}
\boldsymbol{I} \\
\boldsymbol{\alpha}_{L}^{-+}
\end{array}\right) e^{\boldsymbol{U}_{L} \epsilon}(\boldsymbol{I} \otimes \boldsymbol{e}) \boldsymbol{\beta}^{+-} e^{D \epsilon}\right]\right)^{-1} \\
& \times\left(\begin{array}{cc}
\epsilon^{-1} \boldsymbol{I} & \mathbf{0} \\
\mathbf{0} & \boldsymbol{I}
\end{array}\right)\left(\boldsymbol{e}-(\boldsymbol{I} \otimes \boldsymbol{\kappa})\left(\begin{array}{c}
\boldsymbol{I} \\
\boldsymbol{\alpha}_{L}^{-+}
\end{array}\right) e^{\boldsymbol{U}_{L} \epsilon} \boldsymbol{e}\right)
\end{aligned}
$$

Now,

$$
\begin{array}{r}
\lim _{\epsilon \rightarrow 0}\left(\begin{array}{cc}
\epsilon^{-1} \boldsymbol{I} & \mathbf{0} \\
\mathbf{0} & \boldsymbol{I}
\end{array}\right)\left(\boldsymbol{e}-\left(\begin{array}{cc}
\boldsymbol{I} \otimes \boldsymbol{\kappa} & \mathbf{0} \\
\mathbf{0} & \boldsymbol{I} \otimes \boldsymbol{\kappa}
\end{array}\right)\left(\begin{array}{c}
\boldsymbol{I} \\
\boldsymbol{\alpha}_{L}^{-+}
\end{array}\right) \boldsymbol{e}^{\boldsymbol{U}_{L} \epsilon} \boldsymbol{e}\right) \\
\quad=\lim _{\epsilon \rightarrow 0}\left(\begin{array}{c}
\epsilon^{-1}\left(\boldsymbol{e}-(\boldsymbol{I} \otimes \boldsymbol{\kappa}) \boldsymbol{e}^{\boldsymbol{U}_{L} \epsilon} \boldsymbol{e}\right) \\
\boldsymbol{e}-(\boldsymbol{I} \otimes \boldsymbol{\kappa}) \boldsymbol{\alpha}_{L}^{-+} e^{\boldsymbol{U}_{L} \epsilon} \boldsymbol{e}
\end{array}\right)=\left(\begin{array}{c}
-(\boldsymbol{I} \otimes \boldsymbol{\kappa}) \boldsymbol{U}_{L} \boldsymbol{e} \\
\boldsymbol{e}-(\boldsymbol{I} \otimes \boldsymbol{\kappa}) \boldsymbol{\alpha}_{L}^{-+} \boldsymbol{e}
\end{array}\right)
\end{array}
$$

Additionally, we have that

$$
\left.\begin{array}{rl}
\lim _{\epsilon \rightarrow 0} & \left(\begin{array}{cc}
\epsilon^{-1} \boldsymbol{I} & \mathbf{0} \\
\mathbf{0} & \boldsymbol{I}
\end{array}\right)\left[\boldsymbol{I}-\left(\begin{array}{cc}
\boldsymbol{I} \otimes \boldsymbol{\kappa} & \mathbf{0} \\
\mathbf{0} & \boldsymbol{I} \otimes \boldsymbol{\kappa}
\end{array}\right)\left(\begin{array}{c}
\boldsymbol{I} \\
\boldsymbol{\alpha}_{L}^{-+}
\end{array}\right) e^{\boldsymbol{U}_{L} \epsilon}\left(\begin{array}{cc}
\boldsymbol{I} \otimes \boldsymbol{e} & \mathbf{0} \\
\mathbf{0} & \boldsymbol{I} \otimes \boldsymbol{e}
\end{array}\right) \boldsymbol{\beta}^{+-} e^{D \epsilon}\right] \\
& =\lim _{\epsilon \rightarrow 0}\left(\begin{array}{cc}
\epsilon^{-1} \boldsymbol{I} & \mathbf{0} \\
\mathbf{0} & \boldsymbol{I}
\end{array}\right)-\left(\begin{array}{c}
\epsilon^{-1}(\boldsymbol{I} \otimes \boldsymbol{\kappa}) e^{\boldsymbol{U}_{L} \epsilon}(\boldsymbol{I} \otimes \boldsymbol{e}) \\
(\boldsymbol{I} \otimes \boldsymbol{\kappa}) \boldsymbol{\alpha}_{L}^{-+}
\end{array} e^{\boldsymbol{U}_{L} \epsilon}(\boldsymbol{I} \otimes \boldsymbol{e})\right.
\end{array}\right)\left(\begin{array}{ll}
\boldsymbol{I} & \mathbf{0}) e^{D \epsilon} \\
& =-\left(\begin{array}{cc}
(\boldsymbol{I} \otimes \boldsymbol{\kappa}) \boldsymbol{U}_{L}(\boldsymbol{I} \otimes \boldsymbol{e})+\boldsymbol{D}^{\sigma \sigma} & \boldsymbol{D}^{\sigma-} \\
(\boldsymbol{I} \otimes \boldsymbol{\kappa}) \boldsymbol{\alpha}_{L}^{-+}(\boldsymbol{I} \otimes \boldsymbol{e}) & -\boldsymbol{I}
\end{array}\right) .
\end{array}\right.
$$

In the equality leading to (16) we used that

$$
\begin{aligned}
(\boldsymbol{I} \otimes \boldsymbol{\kappa}) e^{\boldsymbol{U}_{L} \epsilon}(\boldsymbol{I} \otimes \boldsymbol{e}) & =(\boldsymbol{I} \otimes \boldsymbol{\kappa})(\boldsymbol{I} \otimes \boldsymbol{e})+(\boldsymbol{I} \otimes \boldsymbol{\kappa})\left(\boldsymbol{U}_{L} \epsilon\right)(\boldsymbol{I} \otimes \boldsymbol{e})+o(\epsilon) \\
& =\boldsymbol{I}+(\boldsymbol{I} \otimes \boldsymbol{\kappa})\left(\boldsymbol{U}_{L} \epsilon\right)(\boldsymbol{I} \otimes \boldsymbol{e})+o(\epsilon) .
\end{aligned}
$$

Also, notice that $(\boldsymbol{I} \otimes \boldsymbol{\kappa}) \boldsymbol{U}_{L}(\boldsymbol{I} \otimes \boldsymbol{e})$ is a sub-intensity matrix and $(\boldsymbol{I} \otimes \boldsymbol{\kappa}) \boldsymbol{\alpha}_{L}^{-+}(\boldsymbol{I} \otimes \boldsymbol{e})$ is a subprobability matrix. To verify this, consider $\boldsymbol{U}_{L}$ to be a block matrix of matrices $\left\{\boldsymbol{U}_{i j}\right\}$ of dimension $\ell \times \ell$. Since $\boldsymbol{U}_{i i} \boldsymbol{e}$ is a nonpositive row vector and $\boldsymbol{U}_{i j}(i \neq j)$ has non-negative entries, then

$$
\begin{aligned}
& \left((\boldsymbol{I} \otimes \boldsymbol{\kappa}) \boldsymbol{U}_{L}(\boldsymbol{I} \otimes \boldsymbol{e})\right)_{i i}=\boldsymbol{\kappa} \boldsymbol{U}_{i i} \boldsymbol{e} \leq 0, \text { and } \\
& \left((\boldsymbol{I} \otimes \boldsymbol{\kappa}) \boldsymbol{U}_{L}(\boldsymbol{I} \otimes \boldsymbol{e})\right)_{i j}=\boldsymbol{\kappa} \boldsymbol{U}_{i j} \boldsymbol{e} \geq 0 \text { for } i \neq j .
\end{aligned}
$$

Since $\boldsymbol{U}_{L} \boldsymbol{e}$ is a nonpositive row vector, we also have that

$$
(\boldsymbol{I} \otimes \boldsymbol{\kappa}) \boldsymbol{U}_{L}(\boldsymbol{I} \otimes \boldsymbol{e}) \boldsymbol{e}=(\boldsymbol{I} \otimes \boldsymbol{\kappa})\left(\boldsymbol{U}_{L} \boldsymbol{e}\right) \leq \mathbf{0},
$$

so that $(\boldsymbol{I} \otimes \boldsymbol{\kappa}) \boldsymbol{U}_{L}(\boldsymbol{I} \otimes \boldsymbol{e})$ is indeed a sub-intensity matrix. That $(\boldsymbol{I} \otimes \boldsymbol{\kappa}) \boldsymbol{\alpha}_{L}^{-+}(\boldsymbol{I} \otimes \boldsymbol{e})$ is a subprobability matrix follows from similar arguments. This implies that (16) is the negative of a sub-intensity matrix and thus nonsingular, so that (13) follows. 
Remark 4.17: The approach used to compute the limits in the proof of Theorem 4.16 is somewhat similar to the L'Hospital rule applied to matrices. However, in our case, matrix differentiation is only made in certain rows of the matrices, the ones corresponding to the states in which the Brownian component is active. Otherwise, if one were to apply L'Hospital rule directly, a singular matrix arises and inversion is not possible.

\subsection{Finite time ruin probabilities}

Finite time ruin probabilities have previously been considered in Asmussen et al. (2002) for SparreAndersen models without Brownian components. Adding a Brownian component does not add complexity to the solution and it can be calculated in an entirely similar way by applying the fluid flow arguments and the corresponding iteration scheme.

In the following, we consider the slightly more interesting problem of calculating the Parisian ruin in a finite time-horizon. First, we need to define what finite time-horizon ruin means in the Parisian case.

Definition 4.18: For $n \geq 1$, let $t_{n}=\int_{0}^{d_{n}} \mathbb{1}_{J_{s} \in E_{+}} \mathrm{d} s$ and $t_{n}^{\epsilon}=\int_{0}^{d_{n}^{\epsilon}} \mathbb{1}_{J_{s} \in E_{\sigma}} \mathrm{d} s$. We define the finite time-horizon Parisian ruin prior to time $Z \sim G$ and with clocks $L_{1}, L_{2}, \ldots \sim F$ as the conditional probability, given $J_{0}=i$ and $V_{0}=u$, as

$$
\psi_{i}^{Z, L}(u)= \begin{cases}\mathbb{P}\left(\left\{\bigcup_{n: t_{n}<Z}\left\{L_{n}<h_{n}\right\}\right\} \cup\left\{Z \in \cup_{n}\left(t_{n}, t_{n}+h_{n}\right)\right\}\right) & \text { if } \sigma=0, \\ \lim _{\epsilon \downarrow 0} \mathbb{P}\left(\left\{\bigcup_{n: t_{n}^{\epsilon}<Z}\left\{L_{n}<h_{n}^{\epsilon}\right\}\right\} \cup\left\{Z \in \bigcup_{n}\left(t_{n}^{\epsilon}, t_{n}^{\epsilon}+h_{n}^{\epsilon}\right)\right\}\right) & \text { if } \sigma>0,\end{cases}
$$

i.e. the probability that there is a failed Parisian recovery before time $Z$ or if the process is undergoing a recovery when $Z$ rings. Similarly, we define the cumulative Parisian probability of ruin prior to $Z \sim G$ and with clock $L \sim G$ (given $J_{0}=i, V_{0}=u$ ) by

$$
\phi_{i}^{Z, L}(u)= \begin{cases}\mathbb{P}\left(\left\{L<\sum_{n: t_{n}<Z} h_{n}\right\} \cup\left\{Z \in \cup_{n}\left(t_{n}, t_{n}+h_{n}\right)\right\}\right) & \text { if } \sigma=0, \\ \lim _{\epsilon \downarrow 0} \mathbb{P}\left(\left\{L<\sum_{n: t_{n}^{\epsilon}<Z} h_{n}^{\epsilon}\right\} \cup\left\{Z \in \cup_{n}\left(t_{n}^{\epsilon}, t_{n}^{\epsilon}+h_{n}^{\epsilon}\right)\right\}\right) & \text { if } \sigma>0 .\end{cases}
$$

Now, assume that $Z \sim \operatorname{PH}(\gamma, \Gamma)$. Then, we consider the same fluid flow risk process as for the unlimited time horizon which must be killed at time $Z$. Thus, we may define a fluid flow risk process $\left\{V_{t}^{F}\right\}_{t \geq 0}$ generated by the Markov jump process $\left\{J_{t}^{Z}\right\}_{t \geq 0}$ with intensity matrix

$$
\boldsymbol{\Lambda}_{Z}=\left(\begin{array}{ccc}
\boldsymbol{\Lambda}^{\sigma \sigma} \oplus \boldsymbol{\Gamma} & \boldsymbol{\Lambda}^{\sigma+} \otimes \boldsymbol{I} & \boldsymbol{\Lambda}^{\sigma-} \otimes \boldsymbol{I} \\
\boldsymbol{\Lambda}^{+\sigma} \otimes \boldsymbol{I} & \boldsymbol{\Lambda}^{++} \oplus \boldsymbol{\Gamma} & \boldsymbol{\Lambda}^{+-} \otimes \boldsymbol{I} \\
\boldsymbol{\Lambda}^{-\sigma} \otimes \boldsymbol{I} & \boldsymbol{\Lambda}^{-+} \otimes \boldsymbol{I} & \boldsymbol{\Lambda}^{--} \otimes \boldsymbol{I}
\end{array}\right)
$$

The matrices $\left(\boldsymbol{\beta}_{Z}^{+-}, \boldsymbol{D}_{Z}, \boldsymbol{\alpha}_{Z, L}^{-+}, \boldsymbol{U}_{Z, L}\right)$ of the ladder processes corresponding to $\boldsymbol{\Lambda}_{Z}$ can be computed in the usual fashion using Subsections 4.1 and 4.2.

Theorem 4.19: (1) Conditional on $J_{0}=i$, the finite time-horizon probability of ruin prior to $Z$ is given by

$$
\left(\boldsymbol{e}_{i}^{\prime} \otimes \boldsymbol{\gamma}\right) \boldsymbol{\beta}_{Z}^{+-} e^{D_{Z} u} \boldsymbol{e}
$$


(2) The probability $\psi_{i}^{Z, L}(u)$ of finite time-horizon Parisian ruin prior to time $Z$ and with Fdistributed clocks $L_{1}, L_{2}, \ldots$ is given by

$$
\left(\boldsymbol{e}_{i}^{\prime} \otimes \boldsymbol{\gamma}\right) \boldsymbol{\beta}_{Z}^{+-} e^{D_{Z} u}\left(\boldsymbol{I}-(\boldsymbol{I} \otimes \boldsymbol{\kappa}) \boldsymbol{\alpha}_{Z, L}^{-+}(\boldsymbol{I} \otimes \boldsymbol{e}) \boldsymbol{\beta}_{Z}^{+-}\right)^{-1}\left(\boldsymbol{e}-\boldsymbol{\alpha}_{Z, L}^{-+} \boldsymbol{e}\right)
$$

if $\sigma=0$, and

$$
\left(\boldsymbol{e}_{i}^{\prime} \otimes \boldsymbol{\gamma}\right) \boldsymbol{\beta}_{Z}^{+-} e^{\boldsymbol{D}_{Z} u}\left(\begin{array}{cc}
-\boldsymbol{D}_{\sigma \sigma}^{Z}-(\boldsymbol{I} \otimes \boldsymbol{\kappa}) \boldsymbol{U}_{Z, L}(\boldsymbol{I} \otimes \boldsymbol{e}) & -\boldsymbol{D}_{\sigma-}^{Z} \\
-(\boldsymbol{I} \otimes \boldsymbol{\kappa}) \boldsymbol{\alpha}_{Z, L}^{-+}(\boldsymbol{I} \otimes \boldsymbol{e}) & \boldsymbol{I}
\end{array}\right)^{-1}\left(\begin{array}{c}
-(\boldsymbol{I} \otimes \boldsymbol{\kappa}) \boldsymbol{U}_{Z, L} \boldsymbol{e} \\
\boldsymbol{e}-(\boldsymbol{I} \otimes \boldsymbol{\kappa}) \boldsymbol{\alpha}_{Z, L}^{-+} \boldsymbol{e}
\end{array}\right)
$$

if $\sigma>0$.

(3) The probability $\phi_{i}^{Z, L}(u)$ of finite time-horizon cumulative Parisian ruin prior to time $Z$ and with F-distributed clock $L$ is given by

$$
\left(\left(\boldsymbol{e}_{i}^{\prime} \otimes \boldsymbol{\gamma}\right) \boldsymbol{\beta}_{Z}^{+-} e^{\boldsymbol{D}_{Z} u} \otimes \boldsymbol{\kappa}\right)\left(\boldsymbol{I}-\boldsymbol{\alpha}_{Z, L}^{-+}\left(\boldsymbol{\beta}_{Z}^{+-} \otimes \boldsymbol{I}\right)\right)^{-1}\left(\boldsymbol{e}-\boldsymbol{\alpha}_{Z, L}^{-+} \boldsymbol{e}\right)
$$

if $\sigma=0$, and

$$
\left(\left(\boldsymbol{e}_{i}^{\prime} \otimes \boldsymbol{\gamma}\right) \boldsymbol{\beta}_{Z}^{+-} e^{\boldsymbol{D}_{Z} u} \otimes \boldsymbol{\kappa}\right)\left(\begin{array}{cc}
-\boldsymbol{U}_{Z, L}-\boldsymbol{D}_{Z}^{\sigma \sigma} \otimes \boldsymbol{I} & -\boldsymbol{D}_{Z}^{\sigma-} \otimes \boldsymbol{I} \\
-\boldsymbol{\alpha}_{Z, L}^{-} & \boldsymbol{I}
\end{array}\right)^{-1}\left(\begin{array}{c}
-U_{Z, L} \boldsymbol{e} \\
\boldsymbol{e}-\boldsymbol{\alpha}_{Z, L}^{-+} \boldsymbol{e}
\end{array}\right)
$$

if $\sigma>0$.

Proof: Item (4.19) follows directly from the construction of $\boldsymbol{\Lambda}_{Z}$. Now, let $\sigma=0$. In a possible subexcursion below 0 , say in the $n$-th one, the defect of the matrix $\boldsymbol{\alpha}_{Z, L}^{-+}$corresponds to the event in which either $L_{n}$ or $Z$ finalizes during the $n$-th sub-excursion below 0 . The defect of the matrix $\boldsymbol{\beta}_{Z}^{+-}$ corresponds to the case in which $Z$ rings while the risk process is above level 0 . Thus,

$$
\begin{aligned}
\mathbb{P} & \left(\cap_{i<n}\left\{L_{i}>h_{i}, Z>t_{i}\right\} \cap\left(\left\{L_{n}<h_{n}\right\} \cup\left\{Z \in\left(t_{n}, t_{n}+h_{n}\right)\right\}\right)\right) \\
& =\left(\boldsymbol{e}_{i}^{\prime} \otimes \boldsymbol{\gamma}\right) \boldsymbol{\beta}_{Z}^{+-} e^{\boldsymbol{D}_{Z} u}\left((\boldsymbol{I} \otimes \boldsymbol{\kappa}) \boldsymbol{\alpha}_{Z, L}^{-+}(\boldsymbol{I} \otimes \boldsymbol{e}) \boldsymbol{\beta}_{Z}^{+-}\right)^{n-1}\left(\boldsymbol{e}-\boldsymbol{\alpha}_{Z, L}^{-+} \boldsymbol{e}\right) .
\end{aligned}
$$

Summing over $n \geq 1$ gives us (4.19) for $\sigma=0$. The case $\sigma>0$ as well as the finite time-horizon cumulative probability of ruin (with both $\sigma=0$ and $\sigma>0$ ) follow from analogous arguments.

\section{Erlangization for Parisian ruin}

Most of the literature on Parisian ruin studies cases in which the clocks are deterministic, say, of fixed length $T>0$. Unfortunately, Theorem 4.6 does not provide a direct solution to this problem, but the following Erlangization scheme provides a natural way to approximate such a solution, to be refined later into Equation (19). Consider clocks $L^{(n)}$, where

$$
L^{(n)} \sim \operatorname{Er}_{n}(n / T)
$$

Chebyshev's inequality implies that $L^{(n)}$ converges in probability to $T$ as $n \rightarrow \infty$. With arguments entirely similar to the proof of formula (15) in Asmussen et al. (2002), or by applying Theorem 11.4.3 of Bladt \& Nielsen (2017), we obtain that

$$
\bar{\psi}_{i j}\left(L^{(n)}\right)=\bar{\psi}_{i j}(T)+\frac{C_{i j}}{n}+O\left(1 / n^{2}\right)
$$

for some fixed $C_{i j} \in \mathbb{R}$, or in matrix notation

$$
\overline{\boldsymbol{\Psi}}\left(L^{(n)}\right)=\overline{\mathbf{\Psi}}(T)+\frac{C}{n}+O\left(1 / n^{2}\right),
$$

where $C=\left\{C_{i j}\right\}$. In the following, we prove that a similar relation carries over to Parisian ruin probabilities. 
Theorem 5.1: For some constant $\gamma \in \mathbb{R}$, we have that

$$
\psi_{i}^{L^{(n)}}(u)=\psi_{i}^{T}(u)+\frac{\gamma}{n}+O\left(1 / n^{2}\right)
$$

In particular, we obtain the Richardson extrapolation formula

$$
\psi_{i}^{T}(u)=(n+1) \psi_{i}^{L^{(n+1)}}(u)-n \psi_{i}^{L^{(n)}}(u)+O(1 / n)
$$

as $n \rightarrow \infty$.

Proof: From the well-known formula, $\left(A_{1}-A_{2} A_{3} A_{4}\right)^{-1}=A_{1}^{-1}+A_{1}^{-1}\left(A_{4}-A_{3} A_{1}^{-1} A_{2}\right)^{-1} A_{1}^{-1}$ we get that

$$
\begin{aligned}
& \left(\boldsymbol{I}-\overline{\boldsymbol{\Psi}}\left(L^{(n)}\right) \boldsymbol{\beta}^{+-}\right)^{-1}=\left(\boldsymbol{I}-\overline{\boldsymbol{\Psi}}(T) \boldsymbol{\beta}^{+-}-\frac{\boldsymbol{C} \boldsymbol{\beta}^{+-}}{n}+O\left(1 / n^{2}\right)\right)^{-1} \\
& =\left(\boldsymbol{I}-\overline{\boldsymbol{\Psi}}(T) \boldsymbol{\beta}^{+-}\right)^{-1}+\left(\boldsymbol{C} \boldsymbol{\beta}^{+-}+\frac{1}{n}\right)\left(\boldsymbol{I}-\overline{\boldsymbol{\Psi}}(T) \boldsymbol{\beta}^{+-}\right)^{-1}+O\left(1 / n^{2}\right)
\end{aligned}
$$

Using Theorem 4.5, and letting $\boldsymbol{A}=\left(I-\overline{\boldsymbol{\Psi}}(T) \boldsymbol{\beta}^{+-}\right)^{-1}$, we then get that

$$
\begin{aligned}
\psi_{i}^{L^{(n)}}(u) & =\boldsymbol{e}_{i}^{\prime} \boldsymbol{\beta}^{+-} e^{\boldsymbol{D} u}\left(\boldsymbol{I}-\overline{\boldsymbol{\Psi}}\left(L^{(n)}\right) \boldsymbol{\beta}^{+-}\right)^{-1}\left(\boldsymbol{e}-\overline{\boldsymbol{\Psi}}\left(L^{(n)}\right) \boldsymbol{e}\right) \\
& =\boldsymbol{e}_{i}^{\prime} \boldsymbol{\beta}^{+-} e^{\boldsymbol{D} u}\left(\boldsymbol{A}+\frac{\boldsymbol{A} \boldsymbol{C} \boldsymbol{\beta}^{+-} \boldsymbol{A}}{n}\right)\left(\boldsymbol{e}-\left(\overline{\boldsymbol{\Psi}}(T)+\frac{\boldsymbol{C}}{n}\right) \boldsymbol{e}\right)+O\left(1 / n^{2}\right)
\end{aligned}
$$

from which the first result follows. The Richardson extrapolation formula follows from a simple manipulation.

While $\psi_{i}^{L^{(n)}}(u) \rightarrow \psi_{i}^{T}(u)$ as $n \rightarrow \infty$, the Richardson extrapolation offers an improved rate of convergence as we will see in Example 7.3, where we study the use approximation of the probability of Parisian ruin with non-stochastic clocks. For the case of cumulative Parisian ruin, a similar result follows directly from Theorem 11.4.3 of Bladt \& Nielsen (2017).

Theorem 5.2: For some constant $\gamma^{\prime} \in \mathbb{R}$, we have that

$$
\phi_{i}^{L^{(n)}}(u)=\phi_{i}^{T}(u)+\frac{\gamma^{\prime}}{n}+O\left(1 / n^{2}\right) .
$$

In particular, we obtain the Richardson extrapolation formula

$$
\phi_{i}^{T}(u)=(n+1) \phi_{i}^{L^{(n+1)}}(u)-n \phi_{i}^{L^{(n)}}(u)+O(1 / n)
$$

as $n \rightarrow \infty$.

\section{Constructing phase-type distributions with given marginals and correlation}

The dependence structures in our risk models will be based on a certain subclass of bivariate phasetype distributions which we construct in this section. More specifically, given a pair of phase-type distributed marginals and a feasible correlation, we will explicitly construct a bivariate phase-type distribution based on Baker's copula (see Baker 2008). The main result of this section is Theorem 6.10, which states that such a class of bivariate phase-type distributions lies within the class $\mathrm{MPH}^{*}$ constructed in Kulkarni (1989). The latter is known to be mathematically tractable and happens to be intimately connected with the theory of fluid flow processes. 


\subsection{The Baker copula}

For a general distribution $F$ and $X_{1}, \ldots, X_{n}$ i.i.d. $\sim F$ we let $X_{i: n}$ denote the $i$-th order statistic among $n$, i.e. $X_{1: n} \leq X_{2: n} \leq \cdots X_{n: n}$, and we let $F_{i: n}$ denote its distribution function. Given two distributions $F$ and $G$, define bivariate distributions $H_{+}$and $H_{-}$with marginals $F$ and $G$ by

$$
H_{+}^{(n)}(x, y)=\frac{1}{n} \sum_{i=1}^{n} F_{i: n}(x) G_{i: n}(y) \text { and } H_{-}^{(n)}(x, y)=\frac{1}{n} \sum_{i=1}^{n} F_{i: n}(x) G_{n-i+1: n}(y) .
$$

The interpretation of $H_{+}^{(n)}$ is the following:

(1) Produce $n$ samples $\left\{X_{i}\right\}_{i \leq n}$ with distribution $F$ and $n$ samples $\left\{Y_{i}\right\}_{i \leq n}$ with distribution $G$.

(2) Order each collection into $\left\{X_{i: n}\right\}_{i \leq n}$ and $\left\{Y_{i: n}\right\}_{i \leq n}$, and pair them according to this ordering.

(3) Select a pair $\left(X_{i: n}, Y_{i: n}\right), i \leq n$ with probability $1 / n$.

The interpretation of $H_{-}^{(n)}$ is similar, but the pairings are done in the opposite direction, that is, they consist of $\left\{\left(X_{i: n}, Y_{n-i+1: n}\right)\right\}_{i \leq n}$. In Lin \& Huang (2010), it is proved (Remark 1) that $H_{+}^{(n)}(x, y)$ maximizes and $H_{-}^{(n)}(x, y)$ minimizes the correlation between the marginals among the family of distributions $H^{(n)}$ which can be written on the form

$$
H^{(n)}(x, y)=\frac{1}{n} \sum_{i=1}^{n} \sum_{j=1}^{n} p_{i j} F_{i: n}(x) G_{j: n}(y)
$$

where $\boldsymbol{P}=\left\{p_{i j}\right\}_{i=1, \ldots, n}$ is a doubly stochastic matrix (i.e. $p_{i j} \geq 0$ and both rows and columns sum to one). If $(X, Y) \sim H^{(n)}$, then the correlation coefficient of $(X, Y)$ is given by

$$
\begin{gathered}
\rho(X, Y)=\frac{\mathbb{E}(X Y)-\mathbb{E}(X) \mathbb{E}(Y)}{\sqrt{\operatorname{Var}(X)} \sqrt{\operatorname{Var}(Y)}} \\
=\frac{1}{\sqrt{\operatorname{Var}(X)} \sqrt{\operatorname{Var}(Y)}}\left(\frac{1}{n} \sum_{i=1}^{n} \sum_{j=1}^{n} p_{i j} \mathbb{E}\left(X_{i: n}\right) \mathbb{E}\left(Y_{j: n}\right)-\mathbb{E}(X) \mathbb{E}(Y)\right) .
\end{gathered}
$$

Remark 6.1: The condition of $\boldsymbol{P}$ being doubly stochastic is needed in order for $H^{(n)}$ to have marginals $F$ and $G$. Indeed,

$$
\begin{gathered}
\lim _{y \rightarrow \infty} H^{(n)}(x, y)=\frac{1}{n} \sum_{i=1}^{n} \sum_{j=1}^{n} p_{i j} F_{i: n}(x)=\frac{1}{n} \sum_{i=1}^{n} F_{i: n}(x)=F(x), \quad \text { and } \\
\lim _{x \rightarrow \infty} H^{(n)}(x, y)=\frac{1}{n} \sum_{j=1}^{n} \sum_{i=1}^{n} p_{i j} G_{j: n}(y)=\frac{1}{n} \sum_{j=1}^{n} G_{j: n}(y)=G(y) .
\end{gathered}
$$

The last equality from (24) (ditto (25)) follows from the following argument: if $X_{1}, \ldots, X_{n} \sim F$ and $U$ is a uniform distribution over $\{1, \ldots, n\}$, then $X_{U: n} \sim F$. 
Remark 6.2: The class of bivariate distributions defined in (21) can be slightly generalized by considering bivariate distributions on the form

$$
H^{(m, n)}(x, y)=\frac{1}{m} \sum_{i=1}^{m} \sum_{j=1}^{n} p_{i j} F_{i: m}(x) G_{j: n}(y)
$$

where $\boldsymbol{P}=\left\{p_{i j}\right\}_{i j}$ is an $m \times n$ non-negative matrix with $\boldsymbol{P} \boldsymbol{e}=\boldsymbol{e}$ and $\boldsymbol{e}^{\prime} \boldsymbol{P}=(m / n) \boldsymbol{e}^{\prime}$. It is straightforward to verify that the properties of Remark 6.1 still hold for this class of distributions and that

$$
\rho(X, Y)=\frac{1}{\sqrt{\operatorname{Var}(X)} \sqrt{\operatorname{Var}(Y)}}\left(\frac{1}{m} \sum_{i=1}^{m} \sum_{j=1}^{n} p_{i j} \mathbb{E}\left(X_{i: m}\right) \mathbb{E}\left(Y_{j: n}\right)-\mathbb{E}(X) \mathbb{E}(Y)\right) .
$$

In Dou et al. (2013), it is further proved that if $F$ and $G$ have finite variances, then $H_{+}^{(n)}(x, y)$ converges to the Fréchet-Hoeffding upper bound $\min (F(x), G(y))$ which is the distribution function with maximal positive correlation $\rho_{\max }$ and corresponds to the comonotonic copula. Notice that $\rho_{\max }$ may well be smaller than 1 if $F$ and $G$ are of different types. By drawing from a mixture of $H_{+}^{(n)}$ and the independent bivariate distribution $F(x) G(y)$, we are able to define a new bivariate distribution that attains any positive feasible correlation coefficient as follows.

Lemma 6.3: Let $F$ and $G$ be distributions with finite variances. Given $0 \leq \rho<\rho_{\max }$. Then there exists an $n \in \mathbb{N}$ and $0 \leq q \leq 1$ such that

$$
\hat{H}^{(n)}(x, y)=q H_{+}^{(n)}(x, y)+(1-q) F(x) G(y)
$$

is a bivariate distribution function with marginals which are correlated with correlation coefficient $\rho$. Moreover,

$$
\hat{H}^{(n)}(x, y)=\frac{1}{n} \sum_{i=1}^{n} \sum_{j=1}^{n}\left(q \delta_{i j}+\frac{1-q}{n}\right) F_{i: n}(x) G_{j: n}(y)
$$

Proof: Since $H_{+}^{(n)}$ converges to the Fréchet-Hoeffding upper limit, there exists an $n$ such that if $(X, Y) \sim H_{+}^{(n)}$, then their correlation coefficient, say $\rho_{+}=\rho(X, Y)$, is contained in the interval $\left(\rho, \rho_{\max }\right)$. In this case let $q=\rho / \rho_{+}$, so that

$$
\mathbb{E}_{\hat{H}^{(n)}}(X Y)=\frac{\rho}{\rho_{+}} \mathbb{E}_{H_{+}^{(n)}}(X Y)+\left(1-\frac{\rho}{\rho_{+}}\right) \mathbb{E}_{F}(X) \mathbb{E}_{G}(Y),
$$

so that if $\left(X_{1}, Y_{1}\right) \sim \hat{H}^{(n)}$ then their correlation coefficient is given by

$$
\rho\left(X_{1}, Y_{1}\right)=\frac{\left[\frac{\rho}{\rho_{+}} \mathbb{E}_{H_{+}^{(n)}}(X Y)+\left(1-\frac{\rho}{\rho_{+}}\right) \mathbb{E}_{F}(X) \mathbb{E}_{G}(Y)\right]-\mathbb{E}_{F}(X) \mathbb{E}_{G}(Y)}{\sqrt{\operatorname{Var}_{F}(X)} \sqrt{\operatorname{Var}_{G}(Y)}}=\frac{\rho}{\rho_{+}} \rho_{+}=\rho .
$$

By (24) and (25),

$$
F(x) G(y)=\frac{1}{n^{2}} \sum_{j=1}^{n} \sum_{j=1}^{n} F_{i: n}(x) G_{j: n}(y)
$$

so that (28) follows. 
Results and constructions concerning negatively correlated random variables are entirely similar, replacing $H_{+}^{(n)}$ with $H_{-}^{(n)}$, approaching the Fréchet-Hoeffding lower bound corresponding to the counter-monotonic copula. Lemma 6.3 thus provides a way of constructing bivariate distributions with given marginals and correlation coefficient based on the distribution of the order statistics of said marginals. We now consider the case where $F$ and $G$ are phase-type distributions.

\subsection{Bivariate phase-type distributions}

Definition 6.4: Let $\tau \sim \mathrm{PH}_{p}(\boldsymbol{\pi}, \boldsymbol{T})$ and let $\left\{J_{t}\right\}_{t \geq 0}$ denote the underlying Markov jump process which generates $\tau$. Let $\boldsymbol{R}=\{r(i, j)\}$ be a $p \times 2$ matrix with non-negative entries. Define

$$
X=\int_{0}^{\tau} r\left(J_{s}, 1\right) \mathrm{d} s \text { and } Y=\int_{0}^{\tau} r\left(J_{s}, 2\right) \mathrm{d} s .
$$

Then $(X, Y)$ is said to have a bivariate phase-type distribution of the $\mathrm{MPH}^{*}$-type (or Kulkarni type) and we write

$$
(X, Y) \sim \mathrm{MPH}^{*}(\boldsymbol{\pi}, \boldsymbol{T}, \boldsymbol{R})
$$

We shall make use of the following notation.

Definition 6.5: Let $\boldsymbol{A}$ be a $m \times n$ matrix and let $\boldsymbol{B}$ be either a $p \times p$ or a $p \times 1$ matrix. For such matrices and $n \geq 1$, define the notation

$$
\begin{aligned}
\boldsymbol{A}^{\otimes n} & =\underbrace{\boldsymbol{A} \otimes \otimes \boldsymbol{A}}_{n \text { terms }} . \\
\boldsymbol{B}^{\oplus n} & =\sum_{j=0}^{n-1} \boldsymbol{I}^{\otimes j} \otimes \boldsymbol{B} \otimes \boldsymbol{I}^{\otimes n-1-j},
\end{aligned}
$$

where $\boldsymbol{I}$ is of dimension $p \times p$.

The following Theorem 6.6, which to our knowledge is new, gives a phase-type representation for the order statistics of phase-type-distributed random variables. This construction was implicitly used in the exponential case in Bladt \& Nielsen (2010). For $n=2$, a similar construction was used in Bladt \& Nielsen (2010) for phase-type and matrix-exponential distributions.

Theorem 6.6: Let $Z_{1}, \ldots, Z_{n}$ i.i.d. $\sim P H(\boldsymbol{\alpha}, \boldsymbol{S}), \boldsymbol{s}=-\boldsymbol{S e}, \boldsymbol{\alpha}_{k: n}=\left(\boldsymbol{\alpha}^{\otimes n}, \mathbf{0}, \mathbf{0}, \ldots, \mathbf{0}\right)$ and

$$
\boldsymbol{S}_{k: n}=\left(\begin{array}{ccccc}
\boldsymbol{S}^{\oplus n} & \boldsymbol{s}^{\oplus(n)} & \mathbf{0} & \cdots & \mathbf{0} \\
\mathbf{0} & \boldsymbol{S}^{\oplus(n-1)} & \boldsymbol{s}^{\oplus(n-1)} & \cdots & \mathbf{0} \\
\vdots & \vdots & \vdots & \ddots & \vdots \\
\mathbf{0} & \mathbf{0} & \mathbf{0} & \cdots & \boldsymbol{S}^{\oplus(n-k+1)}
\end{array}\right) .
$$

Then $Z_{k: n} \sim P H\left(\boldsymbol{\alpha}_{k: n}, \boldsymbol{S}_{k: n}\right)$.

Proof: Consider $n$ independent Markov jump processes $\left\{X_{i}(t)\right\}_{t \geq 0}, i=1, \ldots, n$, underlying $Z_{1}, \ldots, Z_{n}$. The first time a Markov jump process gets absorbed is then phase-type distributed with sub-intensity matrix $S^{\oplus n}=S \oplus \cdots \oplus S$ ( $n$ terms) if the state-space is lexicographically ordered. Hence, $S^{\oplus n}$ is the sub-intensity matrix for the minimum of $Z_{1}, \ldots, Z_{n}$. Killing one process and letting the $n-1$ remaining continue to run, is taken care of by the matrix $\boldsymbol{s}^{\oplus n}$, which makes a transition to a 
smaller state-space where only the $n-1$ remaining processes are present. Repeating this argument $k$ times will provide us with $Z_{k: n}$ and the resulting structure of $S_{k: n}$.

The following provides a recursive formula to compute moments of order statistics of a phase-type distirbution.

Theorem 6.7: Let $Z_{1}, \ldots, Z_{n}$ i.i.d. $\sim P H(\boldsymbol{\alpha}, S)$ and let $\mu_{k: n}=\mathbb{E}\left(Z_{k: n}\right)$. Then

$$
\mu_{k+1: n}=\mu_{k: n}+\boldsymbol{\alpha}^{\otimes n}\left(\prod_{i=0}^{k-1}\left[-S^{\oplus(n-i)}\right]^{-1} \boldsymbol{s}^{\oplus(n-i)}\right)\left(-\boldsymbol{S}^{\oplus(n-k)}\right)^{-1} \boldsymbol{e}
$$

for $k=1, \ldots, n-1$ and where

$$
\mu_{1: n}=\alpha^{\otimes n}\left(-S^{\oplus n}\right)^{-1} \boldsymbol{e}
$$

Proof: Follows easily from Theorem 6.6, $\mu_{k: n}=\mathbb{E}\left(Z_{k: n}\right)=\boldsymbol{\alpha}_{k: n}\left(-S_{k: n}\right)^{-1} \boldsymbol{e}$ and the general block inversion formula

$$
\left(\begin{array}{cc}
\boldsymbol{A} & \boldsymbol{B} \\
\mathbf{0} & \boldsymbol{C}
\end{array}\right)^{-1}=\left(\begin{array}{cc}
\boldsymbol{A}^{-1} & -\boldsymbol{A}^{-1} \boldsymbol{B} \boldsymbol{C}^{-1} \\
\mathbf{0} & \boldsymbol{C}^{-1}
\end{array}\right)
$$

Remark 6.8: There is a probabilistic interpretation of (29): the mean of the $(k+1)$-th order statistic can be decomposed as the mean of the $k$-th order statistic plus the mean remaining time for $Z_{k+1: n}$ to occur.

From the argument at the end of Remark 6.1 and Theorem 6.6, we obtain the following.

Corollary 6.9: Let $n \in \mathbb{N}$ and define

$$
\begin{aligned}
\boldsymbol{\alpha}^{(n)} & =\frac{1}{n}\left(\boldsymbol{\alpha}_{1: n}, \ldots, \boldsymbol{\alpha}_{n: n}\right), \quad \text { and } \\
\boldsymbol{S}^{(n)} & =\left(\begin{array}{cccc}
\boldsymbol{S}_{1: n} & \mathbf{0} & \ldots & \mathbf{0} \\
\mathbf{0} & \boldsymbol{S}_{2: n} & \ldots & \mathbf{0} \\
\vdots & \vdots & \ddots & \vdots \\
\mathbf{0} & \mathbf{0} & \ldots & \boldsymbol{S}_{n: n}
\end{array}\right) .
\end{aligned}
$$

Then $P H\left(\boldsymbol{\alpha}^{(n)}, \boldsymbol{S}^{(n)}\right) \sim P H(\boldsymbol{\alpha}, \boldsymbol{S})$.

We are now able to obtain a bivariate phase-type distribution with given marginals and correlation coefficient using the representation in Corollary 6.9 and Baker's copula.

Theorem 6.10: Let

$$
(X, Y) \sim M P H^{*}\left(\left(\boldsymbol{\alpha}^{(m)}, \mathbf{0}\right),\left(\begin{array}{cc}
\boldsymbol{S}^{(m)} & \boldsymbol{F}^{(m, n)}(\boldsymbol{P}) \\
\mathbf{0} & \boldsymbol{T}^{(n)}
\end{array}\right),\left(\begin{array}{ll}
\boldsymbol{e} & \mathbf{0} \\
\mathbf{0} & \boldsymbol{e}
\end{array}\right)\right),
$$

where

$$
\boldsymbol{F}^{(m, n)}(\boldsymbol{P})=\left\{p_{i j} \boldsymbol{s}_{i: m} \boldsymbol{\beta}_{j: n}\right\}_{(i, j) \in(1, \ldots, m) \times(1, \ldots, n)} .
$$

Then $X \sim P H(\boldsymbol{\alpha}, \boldsymbol{S})$ and $Y \sim P H(\pi, \boldsymbol{T})$ with correlation coefficient given by (27). In particular, any choice of feasible correlation coefficient $\rho$ between $X$ and $Y$ may be attained by choosing a representation (30) with $m=n$, with $n$ and $q$ as in (6.3), and

$$
\boldsymbol{P}=q \boldsymbol{I}+(1-q) \frac{1}{n} \boldsymbol{e} \boldsymbol{e}^{\prime}
$$




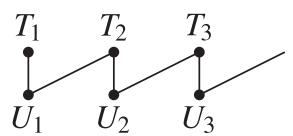

(a) Alternating

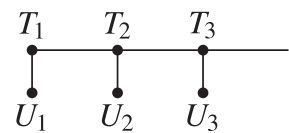

(b) Conditional

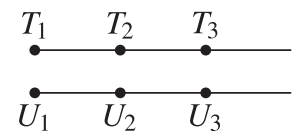

(c) Sequential

Figure 3. (a) Alternating dependence: $U_{i}$ is drawn conditionally on $T_{i}$, and $T_{i+1}$ is drawn conditionally on $U_{i}$; (b) $\left\{U_{i}\right\}$ conditionally independent given a dependent $\left\{T_{i}\right\}$ sequence; (c) sequential independence only: independence between $\left\{T_{i}\right\}$ and $\left\{U_{i}\right\}$ while dependence within each of them.

Proof: Let $E_{X}^{m}$ and $E_{Y}^{n}$ be the phase-space of $\mathrm{PH}\left(\boldsymbol{\alpha}^{(m)}, \boldsymbol{S}^{(m)}\right)$ and $\mathrm{PH}\left(\boldsymbol{\pi}^{(n)}, \boldsymbol{T}^{(n)}\right)$, respectively, and let $\left\{J_{t}\right\}_{t \geq 0}$ be the terminating Markov jump process associated to (30) with state-space $E_{X}^{m} \cup E_{Y}^{n}$. Then

$$
X=\int_{0}^{\infty} \mathbb{1}\left\{J_{s} \in E_{X}^{m}\right\} \mathrm{d} s \quad \text { and } \quad Y=\int_{0}^{\infty} \mathbb{1}\left\{J_{s} \in E_{Y}^{n}\right\} \mathrm{d} s .
$$

From (30), it is clear that

$$
X=\int_{0}^{\tau} \mathbb{1}\left\{J_{s} \in E_{X}^{m}\right\} \mathrm{d} s \quad \text { and } \quad Y=\int_{\tau}^{\infty} \mathbb{1}\left\{J_{s} \in E_{Y}^{n}\right\} \mathrm{d} s=\int_{0}^{\infty} \mathbb{1}\left\{J_{\tau+s} \in E_{Y}^{n}\right\} \mathrm{d} s,
$$

where $\tau=\inf \left\{t>0: J_{t} \in E_{Y}^{n}\right\}$. Thus, by Corollary 6.9, $X \sim \operatorname{PH}(\boldsymbol{\alpha}, \boldsymbol{S})$. Furthermore, the distribution of $J_{\tau}$ is given by

$$
\begin{aligned}
& \int_{0}^{\infty} \boldsymbol{\alpha}^{(m)} e^{\boldsymbol{S}^{(m)} x}\left\{p_{i j} \boldsymbol{s}_{i: m} \boldsymbol{\beta}_{j: n}\right\}_{(i, j) \in(1, \ldots, m) \times(1, \ldots, n)} \mathrm{d} x \\
& \quad=\frac{1}{m}\left(\boldsymbol{\alpha}_{1: m}, \ldots, \boldsymbol{\alpha}_{m: m}\right)\left(\begin{array}{cccc}
-\boldsymbol{S}_{1: m}^{-1} & \mathbf{0} & \ldots & \mathbf{0} \\
\mathbf{0} & -\boldsymbol{S}_{2: m}^{-1} & \ldots & \mathbf{0} \\
\vdots & \vdots & \cdots: . & \vdots \\
\mathbf{0} & \mathbf{0} & \vdots & -\boldsymbol{S}_{m: m}^{-1}
\end{array}\right)\left\{p_{i j} \boldsymbol{s}_{i: m} \boldsymbol{\beta}_{j: n}\right\} \\
& =\frac{1}{m}\left(\sum_{i=1}^{m} p_{i 1} \boldsymbol{\beta}_{1: n}, \cdots, \sum_{i=1}^{m} p_{i n} \boldsymbol{\beta}_{n: n}\right) \\
& =\boldsymbol{\beta}^{(n)}
\end{aligned}
$$

so that by another application of Corollary $6.9, Y \sim \operatorname{PH}(\boldsymbol{\beta}, \boldsymbol{T})$. That $\rho(X, Y)$ is given by (27) follows by noticing that (30) corresponds to the bivariate distribution defined in (21).

\subsection{Examples of dependency structures}

Now that we are able to construct a bivariate phase-type distribution with given marginals and correlation coefficient, we will setup fluid flow risk processes with these characteristics embedded. In particular, we consider four different scenarios: dependence by pairs, alternating dependence (Figure 3(a)), conditional independence (Figure 3(b)) and sequential independence (Figure 3(c)).

Example 6.11: As it was outlined in Section 2, dependence by pairs is the most common in the literature. In this setting, $\left(T_{i}, U_{i}\right)$ is a sequence of i.i.d. bivariate phase-type random variables, whose distribution is given according to (30). Thus, $T_{i} \sim \operatorname{PH}(\boldsymbol{\alpha}, S), U_{i} \sim \operatorname{PH}(\pi, T)$ with some given (feasible) correlation coefficient $\rho\left(T_{i}, U_{i}\right)=\rho$ for all $i \geq 1$. The construction of a fluid flow risk process with these characteristics is as follows. 
Given $m, n \in \mathbb{N}$, let $E(m, n)=(1, \ldots, m) \times(1, \ldots, n)$, let $\boldsymbol{P}=\left\{p_{i j}\right\}_{(i, j) \in E(m, n)}$ be a non-negative matrix such that $\boldsymbol{P} \boldsymbol{e}=\boldsymbol{e}$ and $\boldsymbol{e}^{\prime} \boldsymbol{P}=(m / n) \boldsymbol{e}^{\prime}$, and define

$$
\boldsymbol{F}^{(m, n)}(\boldsymbol{P})=\left\{p_{i j} \boldsymbol{s}_{i: m} \boldsymbol{\beta}_{j: n}\right\}_{(i, j) \in E(m, n)} .
$$

Define $\left\{J_{t}\right\}_{t \geq 0}$ with $J_{0} \sim\left(\boldsymbol{\alpha}^{(m)}, \mathbf{0}\right)$ and intensity matrix

$$
\boldsymbol{\Lambda}=\left(\begin{array}{cc}
\boldsymbol{S}^{(m)} & \boldsymbol{F}^{(m, n)}(\boldsymbol{P}) \\
-\boldsymbol{T}^{(n)} \boldsymbol{e} \boldsymbol{\alpha}^{(m)} & \boldsymbol{T}^{(n)}
\end{array}\right) .
$$

Notice that the first inter-arrival time and first claim size $\left(T_{1}, U_{1}\right)$ are jointly drawn from (30). Once the first claim size finalizes, it is evident from the transition $-\boldsymbol{T}^{(n)} \boldsymbol{e} \boldsymbol{\alpha}^{(m)}$ that $\left(T_{2}, U_{2}\right)$ is drawn independently from $\left(T_{1}, U_{1}\right)$ and $T_{2} \sim \operatorname{PH}\left(\boldsymbol{\alpha}^{(m)}, \boldsymbol{S}^{(m)}\right)$. Recursively, we end up with a sequence $\left(T_{i}, U_{i}\right)_{i \geq 1}$ of independent vectors with bivariate distribution given by (31). In order to achieve that $\rho\left(T_{i}, U_{i}\right)=\rho$ for all $i \geq 1$, it is enough to take $m, n$ and $\boldsymbol{P}$ as in Theorem 6.10.

The following kind of dependencies, although more involved, are straightforward extensions of the dependence by pairs model. Finding explicit solutions for the ruin probabilities of these models is not possible through classical methods. We believe that this demonstrates the flexibility that risk modelling within the fluid flow framework can achieve.

Example 6.12: Given $m, n \in \mathbb{N}$, let $\boldsymbol{P}$ and $\boldsymbol{F}^{(m, n)}(\boldsymbol{P})$ be as in Example 6.11, let $\boldsymbol{Q}=\left\{q_{i j}\right\}_{(i, j) \in E(n, m)}$ be a non-negative matrix such that $\boldsymbol{Q} \boldsymbol{e}=\boldsymbol{e}$ and $\boldsymbol{e}^{\prime} \boldsymbol{Q}=(n / m) \boldsymbol{e}^{\prime}$, and define

$$
\boldsymbol{G}^{(n, m)}(\boldsymbol{Q})=\left\{q_{i j} \boldsymbol{t}_{i: n} \boldsymbol{\alpha}_{j: m}\right\}_{(i, j) \in E(n, m)} .
$$

Define $\left\{J_{t}\right\}_{t \geq 0}$ with $J_{0} \sim\left(\boldsymbol{\alpha}^{(m)}, \mathbf{0}\right)$ and intensity matrix

$$
\boldsymbol{\Lambda}=\left(\begin{array}{cc}
\boldsymbol{S}^{(m)} & \boldsymbol{F}^{(m, n)}(\boldsymbol{P}) \\
\boldsymbol{G}^{(n, m)}(\boldsymbol{Q}) & \boldsymbol{T}^{(n)}
\end{array}\right)
$$

Just as in Example 6.11, $\left(T_{1}, U_{1}\right)$ has a distribution given by (30), however, $\left(U_{1}, T_{2}\right)$ now has a distribution given by

$$
\mathrm{MPH}^{*}\left(\left(\mathbf{0}, \boldsymbol{\beta}^{(m)}\right),\left(\begin{array}{cc}
\boldsymbol{S}^{(m)} & \mathbf{0} \\
\boldsymbol{G}^{(n, m)}(\boldsymbol{Q}) & \boldsymbol{T}^{(n)}
\end{array}\right),\left(\begin{array}{ll}
\boldsymbol{e} & \mathbf{0} \\
\mathbf{0} & \boldsymbol{e}
\end{array}\right)\right)
$$

This way we further introduce dependence (and thus correlation) between $U_{i}$ and $T_{i+1}$ for all $i \geq 1$ while keeping the same dependence structure between $T_{i}$ and $U_{i}$ described in Example 6.11 and fixed marginals for $\left\{T_{i}\right\}$ and $\left\{U_{i}\right\}$. In order to achieve fixed feasible correlation coefficients $\rho\left(T_{i}, U_{i}\right)=\rho_{1}$ and $\rho\left(U_{i}, T_{i+1}\right)=\rho_{2}$ for all $i \geq 1$, it is enough to chose sufficiently large $n$ and $m$ as in Theorem 6.10, $\boldsymbol{P}$ as in Theorem 6.10 with $\rho$ replaced by $\rho_{1}$, and $\boldsymbol{Q}$ as $\boldsymbol{P}$ in Theorem 6.10 with $\rho$ replaced by $\rho_{2}$.

Example 6.13: The cases which involve conditional independence or complete independence between the sequences are more involved since we need to keep track of the exit state of the dependent variables. First consider the case with $m=n=2$. Let $\boldsymbol{P}$ and $\boldsymbol{Q}$ be doubly stochastic $2 \times 2$ matrices and let $\left\{J_{t}\right\}_{t \geq 0}$ be a Markov jump process with $J_{0} \sim\left(\boldsymbol{\alpha}^{(2)}, \mathbf{0}\right)$ and intensity matrix

$$
\left(\begin{array}{cc|cccc}
\boldsymbol{s}_{1: 2} & \mathbf{0} & p_{11} \boldsymbol{s}_{1: 2} \boldsymbol{\beta}_{1: 2} & \mathbf{0} & p_{12} \boldsymbol{s}_{1: 2} \boldsymbol{\beta}_{2: 2} & \mathbf{0} \\
\mathbf{0} & \boldsymbol{s}_{2: 2} & \mathbf{0} & p_{21} \boldsymbol{s}_{2: 2} \boldsymbol{\beta}_{1: 2} & \mathbf{0} & p_{22} \boldsymbol{s}_{2: 2} \boldsymbol{\beta}_{2: 2} \\
\hline q_{11} \boldsymbol{t}_{1: 2} \boldsymbol{\alpha}_{1: 2} & q_{12} \boldsymbol{t}_{1: 2} \boldsymbol{\alpha}_{2: 2} & \boldsymbol{T}_{1: 2} & \mathbf{0} & \mathbf{0} & \mathbf{0} \\
q_{21} \boldsymbol{t}_{1: 2} \boldsymbol{\alpha}_{1: 2} & q_{22} \boldsymbol{t}_{1: 2} \boldsymbol{\alpha}_{2: 2} & \mathbf{0} & \boldsymbol{T}_{1: 2} & \mathbf{0} & \mathbf{0} \\
q_{11} \boldsymbol{t}_{2: 2} \boldsymbol{\alpha}_{1: 2} & q_{12} \boldsymbol{t}_{2: 2} \boldsymbol{\alpha}_{2: 2} & \mathbf{0} & \mathbf{0} & \boldsymbol{T}_{2: 2} & \mathbf{0} \\
q_{21} \boldsymbol{t}_{2: 2} \boldsymbol{\alpha}_{1: 2} & q_{22} \boldsymbol{t}_{2: 2} \boldsymbol{\alpha}_{2: 2} & \mathbf{0} & \mathbf{0} & \mathbf{0} & \boldsymbol{T}_{2: 2}
\end{array}\right) .
$$

Draw $T_{1}$ according to $\left(\boldsymbol{\alpha}^{(2)}, \boldsymbol{S}^{(2)}\right)$. This is done in the usual way of Section 6 but instead of making the transition from a $2 \times 2$ block matrix to another $2 \times 2$ block matrix, we make a transition to a 


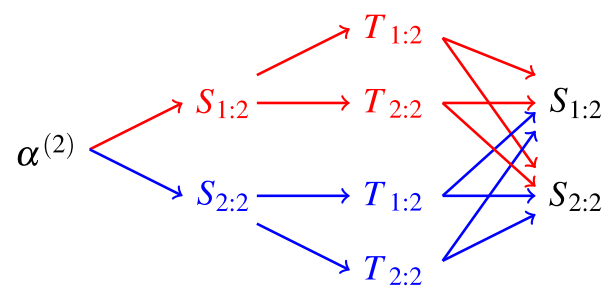

Figure 4. Possible paths of the Markov jump process $\left\{J_{t}\right\}_{t \geq 0}$ through the states in the indicated matrices.

$4 \times 4$ block matrix as shown. We draw $U_{1}$ which depends on $T_{1}$, but when proceeding to draw $T_{2}$ we see that this will be drawn independently of $U_{1}$ and dependent on $T_{1}$. Indeed, regardless of coming from a state in $\boldsymbol{T}_{1: 2}$ or in $\boldsymbol{T}_{2: 2}$, the probability of going to a state in $S_{1: 2}$ is the same (similar for the blue track), however, the drawing of $T_{2}$ does depend on the colour if $q_{11} \neq q_{21}$ and therefore on the original chosen colour through $T_{1}$. Hence $T_{1}$ and $T_{2}$ are dependent, $U_{1}$ depends on $T_{1}$, and $T_{2}$ is conditionally independent of $U_{1}$ given $T_{1}$ (Figure 4 ).

A small reflection shows that the extension to general $\boldsymbol{P}$ and $\boldsymbol{Q}$ matrices is given by (indices 'ci' for conditional independence)

$$
\boldsymbol{\Lambda}=\left(\begin{array}{cc}
\boldsymbol{S}^{(m)} & \boldsymbol{F}_{\mathrm{ci}}^{(m, n)}(\boldsymbol{P}) \\
\boldsymbol{G}_{\mathrm{ci}}^{(n, m)}(\boldsymbol{Q}) & \boldsymbol{T}_{\mathrm{ci}}^{(n)}
\end{array}\right),
$$

where $\boldsymbol{\alpha}_{\mathrm{ci}}^{(m)}=\left(\boldsymbol{\alpha}^{(m)}, \mathbf{0}\right), \boldsymbol{T}_{\mathrm{ci}}^{(n)}=\boldsymbol{\Delta}\left(\left\{\boldsymbol{I}^{(m)} \otimes \boldsymbol{T}_{i: n}\right\}_{i=1, \ldots, n}\right)$,

$$
\begin{aligned}
& \boldsymbol{F}_{\mathrm{ci}}^{(m, n)}(\boldsymbol{P})=\left(\begin{array}{llll}
\boldsymbol{\Delta}\left(\left\{p_{i 1} \boldsymbol{s}_{i: m} \boldsymbol{\beta}_{1: n}\right\}_{i=1, \ldots, m}\right) & \cdots & \boldsymbol{\Delta}\left(\left\{p_{i n} \boldsymbol{s}_{i: m} \boldsymbol{\beta}_{n: n}\right\}_{i=1, \ldots, m}\right)
\end{array}\right), \\
& \left.\boldsymbol{G}_{\mathrm{ci}}^{(n, m)}(\boldsymbol{Q})=\left(\begin{array}{ccc}
q_{11} \boldsymbol{t}_{1: n} \boldsymbol{\alpha}_{1: m} & \cdots & q_{1 m} \boldsymbol{t}_{1: n} \boldsymbol{\alpha}_{m: m} \\
\vdots & \ddots & \vdots \\
q_{n 1} \boldsymbol{t}_{1: n} \boldsymbol{\alpha}_{1: m} & \cdots & q_{n m} \boldsymbol{t}_{1: n} \boldsymbol{\alpha}_{m: m}
\end{array}\right)\right)
\end{aligned}
$$

Here $\boldsymbol{I}^{(m)}$ denotes the $m \times m$-dimensional identity matrix and $\boldsymbol{\Delta}\left(\left\{\boldsymbol{A}_{i}\right\}\right)$ denotes the block-diagonal matrix with block-elements $\left\{\boldsymbol{A}_{i}\right\}$ in its diagonal. In order to achieve fixed and feasible correlation coefficients $\rho\left(T_{i}, U_{i}\right)=\rho_{1}$ and $\rho\left(T_{i}, T_{i+1}\right)=\rho_{2}$ for all $i \geq 1$, it is enough to chose sufficiently large $n, m, \boldsymbol{P}$ and $\boldsymbol{Q}$ as in Example 6.12.

Example 6.14: Again, the case $m=n=2$ is instructive. By arguments similar to Example 6.13, we see that the following matrix

$\left(\begin{array}{cccc|cccc}\boldsymbol{s}_{1: 2} & \mathbf{0} & \mathbf{0} & \mathbf{0} & p_{11} \boldsymbol{s}_{1: 2} \boldsymbol{\beta}_{1: 2} & \mathbf{0} & p_{12} \boldsymbol{s}_{1: 2} \boldsymbol{\beta}_{2: 2} & \mathbf{0} \\ \mathbf{0} & \boldsymbol{S}_{2: 2} & \mathbf{0} & \mathbf{0} & \mathbf{0} & p_{11} \boldsymbol{s}_{2: 2} \boldsymbol{\beta}_{1: 2} & \mathbf{0} & p_{12} \boldsymbol{s}_{2: 2} \boldsymbol{\beta}_{2: 2} \\ \mathbf{0} & \mathbf{0} & \boldsymbol{S}_{1: 2} & \mathbf{0} & p_{21} \boldsymbol{s}_{1: 2} \boldsymbol{\beta}_{1: 2} & \mathbf{0} & p_{22} \boldsymbol{s}_{1: 2} \boldsymbol{\beta}_{2: 2} & \mathbf{0} \\ \mathbf{0} & \mathbf{0} & \mathbf{0} & \boldsymbol{S}_{2: 2} & \mathbf{0} & p_{21} \boldsymbol{s}_{2: 2} \boldsymbol{\beta}_{1: 2} & \mathbf{0} & p_{22} \boldsymbol{s}_{2: 2} \boldsymbol{\beta}_{2: 2} \\ \hline q_{11} \boldsymbol{t}_{1: 2} \boldsymbol{\alpha}_{1: 2} & q_{12} \boldsymbol{t}_{1: 2} \boldsymbol{\alpha}_{2: 2} & \mathbf{0} & \mathbf{0} & \boldsymbol{T}_{1: 2} & \mathbf{0} & \mathbf{0} & \mathbf{0} \\ q_{21} \boldsymbol{t}_{1: 2} \boldsymbol{\alpha}_{1: 2} & q_{22} \boldsymbol{t}_{1: 2} \boldsymbol{\alpha}_{2: 2} & \mathbf{0} & \mathbf{0} & \mathbf{0} & \boldsymbol{T}_{1: 2} & \mathbf{0} & \mathbf{0} \\ \mathbf{0} & \mathbf{0} & q_{11} \boldsymbol{t}_{2: 2} \boldsymbol{\alpha}_{1: 2} & q_{12} \boldsymbol{t}_{2: 2} \boldsymbol{\alpha}_{2: 2} & \mathbf{0} & \mathbf{0} & \boldsymbol{T}_{2: 2} & \mathbf{0} \\ \mathbf{0} & \mathbf{0} & q_{21} \boldsymbol{t}_{2: 2} \boldsymbol{\alpha}_{1: 2} & q_{22} \boldsymbol{t}_{2: 2} \boldsymbol{\alpha}_{2: 2} & \mathbf{0} & \mathbf{0} & \mathbf{0} & \boldsymbol{T}_{2: 2}\end{array}\right)$,


will maintain independent draws between $T_{i}$ 's and $U_{i}$ 's, but that the block structure implies that $T_{i+1}$ does depend on $T_{i}$ and $U_{i+1}$ on $U_{i}$. The initial distribution is set to

$$
1 / 4\left(\boldsymbol{\alpha}_{1: 2}, \boldsymbol{\alpha}_{2: 2}, \boldsymbol{\alpha}_{1: 2}, \boldsymbol{\alpha}_{2: 2}, \mathbf{0} \ldots, \mathbf{0}\right) .
$$

Extension to the $n, m$-case is given by

$$
\boldsymbol{\Lambda}=\left(\begin{array}{cc}
\boldsymbol{S}_{\mathrm{sq}}^{(m)} & \boldsymbol{F}_{\mathrm{sq}}^{(m, n)}(\boldsymbol{P}) \\
\boldsymbol{G}_{\mathrm{sq}}^{(n, m)}(\boldsymbol{Q}) & \boldsymbol{T}_{\mathrm{sq}}^{(n)}
\end{array}\right)
$$

with $\boldsymbol{\alpha}_{\mathrm{Sq}}^{(m)}=1 / n\left(\boldsymbol{e}^{(n)^{\prime}} \otimes \boldsymbol{\alpha}^{(m)}, \mathbf{0}\right), \boldsymbol{S}_{\mathrm{Sq}}^{(m)}=\boldsymbol{I}^{(n)} \otimes \boldsymbol{S}^{(m)}, \boldsymbol{T}_{\mathrm{sq}}^{(n)}=\boldsymbol{\Delta}\left(\left\{\boldsymbol{I}^{(m)} \otimes \boldsymbol{T}_{i: n}\right\}_{i=1, \ldots, n}\right)$,

$$
\boldsymbol{F}_{\mathrm{Sq}}^{(m, n)}(\boldsymbol{P})=\left(\begin{array}{ccc}
\boldsymbol{\Delta}\left(\left\{p_{11} \boldsymbol{s}_{i: m} \boldsymbol{\beta}_{1: n}\right\}_{i=1, \ldots, m}\right) & \cdots & \boldsymbol{\Delta}\left(\left\{p_{1 n} \boldsymbol{s}_{i: m} \boldsymbol{\beta}_{n: n}\right\}_{i=1, \ldots, m}\right) \\
\vdots & \ddots & \vdots \\
\boldsymbol{\Delta}\left(\left\{p_{n 1} \boldsymbol{s}_{i: m} \boldsymbol{\beta}_{1: n}\right\}_{i=1, \ldots, m}\right) & \cdots & \boldsymbol{\Delta}\left(\left\{p_{n n} \boldsymbol{s}_{i: m} \boldsymbol{\beta}_{n: n}\right\}_{i=1, \ldots, m}\right)
\end{array}\right)
$$

and

$$
\boldsymbol{G}_{\mathrm{Sq}}^{(n, m)}(\boldsymbol{Q})=\boldsymbol{\Delta}\left(\left\{\left(\begin{array}{ccc}
q_{11} \boldsymbol{t}_{i: n} \boldsymbol{\alpha}_{1: m} & \cdots & q_{1 m} \boldsymbol{t}_{i: n} \boldsymbol{\alpha}_{m: m} \\
\vdots & \ddots & \vdots \\
q_{m 1} \boldsymbol{t}_{i: n} \boldsymbol{\alpha}_{1: m} & \cdots & q_{m m} \boldsymbol{t}_{i: n} \boldsymbol{\alpha}_{m: m}
\end{array}\right)\right\}_{i=1, \ldots, n}\right)
$$

In order to achieve fixed and feasible correlation coefficients $\rho\left(T_{i}, T_{i+1}\right)=\rho_{1}$ and $\rho\left(U_{i}, U_{i+1}\right)=\rho_{2}$ for all $i \geq 1$, it is enough to chose sufficiently large $n, m$, and $\boldsymbol{P}$ and $\boldsymbol{Q}$ as in Example 6.12.

\section{Numerical examples}

In the following, we study several aspects of our model. In Example 7.1, we make an exhaustive study of how dependency affects the infinite time-horizon probability of ruin of a risk reserve process with exponentially distributed inter-arrival times and claim sizes. In Example 7.2, we test the robustness of our model, in the sense that if certain correlation can be achieved with, say, $n_{0}$ order statistics, then it will also be possible to model the same correlation with $n$ order statistics for all $n>n_{0}$, each one with different ruin probabilities. In Example 7.3, we study how well the erlangization method approximates the case of Parisian ruin with deterministic clocks for a Sparre-Andersen process. Finally, in Example 7.4, we compute the infinite time-horizon, Parisian and cumulative Parisian probability of ruin for a dependent Sparre-Andersen process with or without Brownian noise.

Example 7.1 (The effect of dependency): In order to study the effect of the dependency between inter-arival times and claim sizes, we consider the simplest possible model where both aforementioned random variables are exponentially distributed. To be specific, we let $\sigma=0$ and standardize the inter-arrival times to be exponentially distributed with intensity 1, claim sizes exponentially distributed with intensity 1.2. The completely independent case corresponds to the classical Cramér-Lundberg process with exponential claim sizes. We study the effect correlations have on the infinite time-horizon ruin probabilities for the alternating and sequential dependency structures of Examples 6.12 and 6.14. The reason we chose to work with exponentially distributed components in this example is to strip all other complexities of the process to the bare minimum. This will put in evidence the considerable effect that introducing dependence has, even in the simplest model possible.

For the alternating case, we employ the construction from Example 6.12 based on $m=n=10$ (number of order statistics). This allows for studying correlations in the interval $(-0.55,0.71)$ which 
Table 1. Probability of ruin for the alternating dependent model when $u=V_{0}=0$.

\begin{tabular}{lccccccrr}
\hline & \multicolumn{7}{c}{$\rho\left(U_{i}, T_{i+1}\right)$} \\
\cline { 2 - 8 }$\rho\left(T_{i}, U_{i}\right)$ & -0.5 & -0.3 & -0.1 & 0 & 0.1 & 0.3 & 0.5 & 0.7 \\
\hline-0.5 & 0.8909 & 0.8807 & 0.8693 & 0.8629 & 0.8578 & 0.8447 & 0.8253 & 0.7907 \\
-0.3 & 0.8700 & 0.8641 & 0.8571 & 0.8530 & 0.8502 & 0.8435 & 0.8350 & 0.8238 \\
-0.1 & 0.8466 & 0.8446 & 0.8421 & 0.8407 & 0.8399 & 0.8380 & 0.8356 & 0.8325 \\
0 & 0.8333 & 0.8333 & 0.8333 & 0.8333 & 0.8333 & 0.8333 & 0.8333 & 0.8333 \\
0.1 & 0.8217 & 0.8222 & 0.8229 & 0.8233 & 0.8238 & 0.8249 & 0.8264 & 0.8284 \\
0.3 & 0.7931 & 0.7942 & 0.7962 & 0.7974 & 0.7989 & 0.8024 & 0.8073 & 0.8148 \\
0.5 & 0.7529 & 0.7542 & 0.7571 & 0.7592 & 0.7613 & 0.7667 & 0.7748 & 0.7899 \\
0.7 & 0.6860 & 0.6924 & 0.6955 & 0.6972 & 0.6982 & 0.7003 & 0.7021 & 0.6971 \\
\hline
\end{tabular}

Table 2. Probability of ruin for the alternating dependent model when $u=V_{0}=10$.

\begin{tabular}{lccccccrr}
\hline & \multicolumn{7}{c}{$\rho\left(U_{i}, T_{i+1}\right)$} \\
\cline { 2 - 8 }$\rho\left(T_{i}, U_{i}\right)$ & -0.5 & -0.3 & -0.1 & 0 & 0.1 & 0.3 & 0.5 & 0.7 \\
\hline-0.5 & 0.7390 & 0.5174 & 0.3184 & 0.2307 & 0.1702 & 0.0762 & 0.0225 & 0.0041 \\
-0.3 & 0.5073 & 0.3731 & 0.2444 & 0.1850 & 0.1420 & 0.0701 & 0.0229 & 0.0029 \\
-0.1 & 0.3033 & 0.2375 & 0.1702 & 0.1370 & 0.1096 & 0.0593 & 0.0213 & 0.0026 \\
0 & 0.2155 & 0.1764 & 0.1344 & 0.1128 & 0.0920 & 0.0522 & 0.0199 & 0.0026 \\
0.1 & 0.1550 & 0.1315 & 0.1041 & 0.0890 & 0.0741 & 0.0442 & 0.0182 & 0.0027 \\
0.3 & 0.0645 & 0.0593 & 0.0511 & 0.0458 & 0.0401 & 0.0274 & 0.0138 & 0.0029 \\
0.5 & 0.0166 & 0.0162 & 0.0152 & 0.0144 & 0.0136 & 0.0113 & 0.0080 & 0.0032 \\
0.7 & 0.0019 & 0.0012 & 0.0011 & 0.0011 & 0.0012 & 0.0013 & 0.0015 & 0.0025 \\
\hline
\end{tabular}

are within the feasible range of $\left(1-\pi^{2} / 6,1\right)=(-0.64,1)$. The dimension of the resulting phasetype representations, corresponding to Corollary 6.9, is all 110. In order to obtain correlations larger than 0.71 or smaller than -0.55 , we should have to increase $n$ and thereby the dimension. Intuitively, positive correlations in the alternating model mean that large claim sizes are commonly followed by large inter-arrival times, so that these upward and downward movements even out with each other and the probability of ruin is reduced. Negative correlations in the alternating model imply that short inter-arrival times are usually followed by large claim sizes, so that the probability of getting ruined is higher. This is confirmed in Table 1 for $u=0$ and in Table 2 for $u=10$. Notice that the dependency of the probability of ruin on the correlation is visibly more meaningful for the case $u=10$ than for $u=0$.

For the sequential case, we use the method of Example 6.14 based on $m=n=5$ order statistics with an implied range of correlations of $(-0.46,0.54)$. The reason for diminishing the range is for computational reasons (dimensionality) since the representations in the sequential construction have the double dimension of the corresponding alternating ones. For the sequential model, having positive correlation means that there will be sequences of large claim sizes, and if those happen at the same time of a sequence of short inter-arrival times, then the probability of getting ruined is high. Negative correlation means that large claim sizes are followed by small claim sizes, so that the process evens out. Both points are confirmed in Table 3 containing ruin probabilities for the case of $u=0$ and Table 4 for the case $u=10$. Just as in the alternating model, for the sequential model, the dependency of the probability of ruin on the correlation is more meaningful when $u=10$ than for $u=0$. For this case, even modest changes in the correlation have quite some impact on the probability of getting ruined.

One of the conclusions we may draw from the present example is that the effect of correlation is opposite in the alternating and sequentially independent cases and therefore that the way we construct the dependency in the model is of substantial importance.

Example 7.2 (Robustness of ruin under different constructions): Here we look at an example of how sensitive the probability of ruin is to the actual construction of the desired dependency. 
Table 3. Probability of ruin for the sequential dependent model when $V_{0}=0$.

\begin{tabular}{lcccccccrr}
\hline & \multicolumn{7}{c}{$\rho\left(U_{i}, U_{i+1}\right)$} \\
\cline { 2 - 9 }$\rho\left(T_{i}, T_{i+1}\right)$ & -0.3 & -0.2 & -0.1 & 0 & 0.1 & 0.2 & 0.3 & 0.4 & 0.5 \\
\hline $\mathbf{0 . 3}$ & 0.8230 & 0.8238 & 0.8245 & 0.8253 & 0.8258 & 0.8263 & 0.8268 & 0.8273 & 0.8277 \\
$-\mathbf{0 . 2}$ & 0.8249 & 0.8253 & 0.8258 & 0.8263 & 0.8267 & 0.8271 & 0.8276 & 0.8282 & 0.8289 \\
$\mathbf{0 . 1}$ & 0.8285 & 0.8287 & 0.8289 & 0.8292 & 0.8294 & 0.8297 & 0.8300 & 0.8303 & 0.8308 \\
$\mathbf{0}$ & 0.8333 & 0.8333 & 0.8333 & 0.8333 & 0.8333 & 0.8333 & 0.8333 & 0.8333 & 0.8333 \\
$\mathbf{0 . 1}$ & 0.8398 & 0.8397 & 0.8395 & 0.8393 & 0.8390 & 0.8385 & 0.8380 & 0.8373 & 0.8363 \\
$\mathbf{0 . 2}$ & 0.8470 & 0.8469 & 0.8466 & 0.8461 & 0.8455 & 0.8448 & 0.8437 & 0.8423 & 0.8399 \\
$\mathbf{0 . 3}$ & 0.8552 & 0.8551 & 0.8548 & 0.8543 & 0.8535 & 0.8524 & 0.8509 & 0.8487 & 0.8447 \\
$\mathbf{0 . 4}$ & 0.8649 & 0.8650 & 0.8648 & 0.8643 & 0.8635 & 0.8624 & 0.8607 & 0.8578 & 0.8517 \\
$\mathbf{0 . 5}$ & 0.8777 & 0.8780 & 0.8782 & 0.8782 & 0.8780 & 0.8775 & 0.8765 & 0.8742 & 0.8666 \\
\hline
\end{tabular}

Table 4. Probability of ruin for the sequential dependent model when $V_{0}=10$.

\begin{tabular}{lcccccccrr}
\hline & \multicolumn{7}{c}{$\rho\left(U_{i}, U_{i+1}\right)$} \\
\cline { 2 - 9 }$\rho\left(T_{i}, T_{i+1}\right)$ & -0.3 & -0.2 & -0.1 & 0 & 0.1 & 0.2 & 0.3 & 0.4 & 0.5 \\
\hline-0.3 & 0.0553 & 0.0562 & 0.0646 & 0.0801 & 0.1059 & 0.1449 & 0.2071 & 0.3143 & 0.5209 \\
-0.2 & 0.0584 & 0.0592 & 0.0677 & 0.0833 & 0.1090 & 0.1479 & 0.2098 & 0.3163 & 0.5219 \\
-0.1 & 0.0684 & 0.0694 & 0.0781 & 0.0941 & 0.1199 & 0.1584 & 0.2193 & 0.3233 & 0.5248 \\
0 & 0.0863 & 0.0874 & 0.0965 & 0.1128 & 0.1385 & 0.1762 & 0.2351 & 0.3351 & 0.5295 \\
0.1 & 0.1129 & 0.1143 & 0.1237 & 0.1401 & 0.1653 & 0.2017 & 0.2577 & 0.3519 & 0.5363 \\
0.2 & 0.1552 & 0.1568 & 0.1661 & 0.1819 & 0.2056 & 0.2393 & 0.2906 & 0.3762 & 0.5463 \\
0.3 & 0.2267 & 0.2284 & 0.2367 & 0.2506 & 0.2709 & 0.2995 & 0.3426 & 0.4148 & 0.5626 \\
0.4 & 0.3566 & 0.3582 & 0.3640 & 0.3735 & 0.3871 & 0.4063 & 0.4354 & 0.4851 & 0.5940 \\
0.5 & 0.6158 & 0.6165 & 0.6182 & 0.6208 & 0.6245 & 0.6297 & 0.6377 & 0.6516 & 0.6858 \\
\hline
\end{tabular}

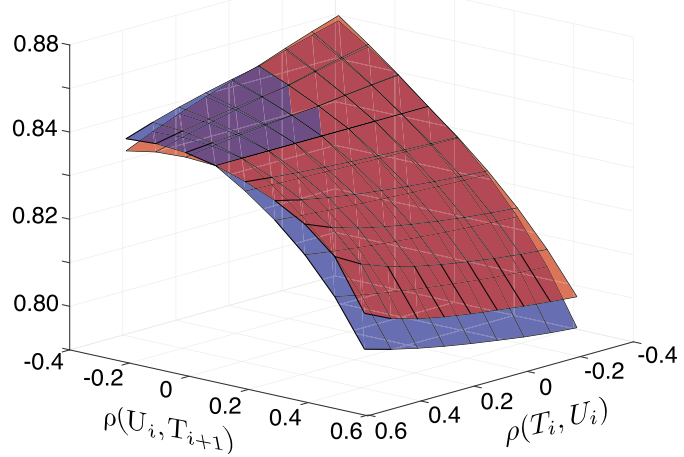

(a)

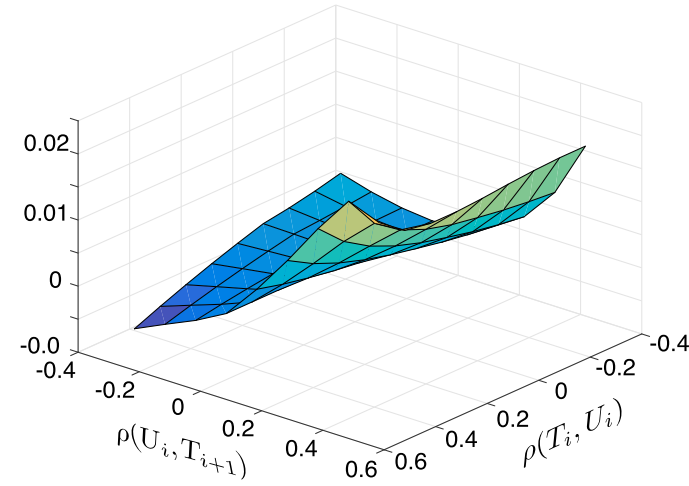

(b)

Figure 5. (a) The surfaces for the ruin probabilities with different correlations for the cases of $m=n=5$ (red) and $m=n=20$ (blue); (b) relative difference between the surfaces (as seen from $m=n=5$ ).

According to Lemma 6.3 and the comments at the end of Section 6, we may attain any feasible correlation coefficient with $n$ order statistics where $n$ is chosen sufficiently large.

We consider the alternating model of Example 7.1, that is, one with exponentially distributed marginals for $U_{i}$ and $T_{i}$, and with pairwise correlations between $T_{i}, U_{i}$ and $U_{i}, T_{i+1}$ in the range of -0.3 to 0.5 . This can be constructed using $m=n=5$ of Example 6.12 but it can also be constructed with, e.g. $m=n=20$. In Figure 5(a), we compare the probability of ruin when $u=0$ and $\rho\left(T_{i}, U_{i}\right), \rho\left(U_{i}, T_{i+1}\right) \in\{-0.3,-0.2, \ldots, 0.5\}$ for the cases where $m=n=5$ and $m=n=20$. In Figure 5(b), we show a plot of the relative difference as seen from $m=n=5$. 

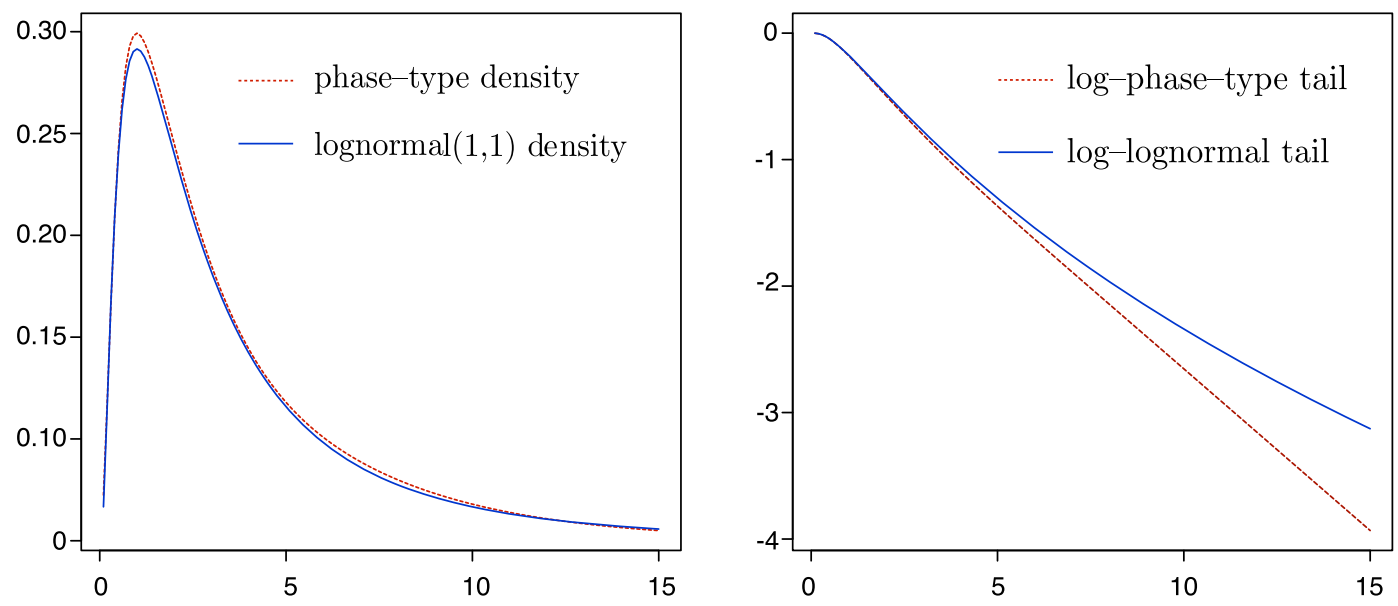

Figure 6. A five-dimensional phase-type fit to the log-normal distribution $\operatorname{LN}(1,1)$. Density comparison in the left figure and comparison of log-survival functions (tails) to the right.

Recall that $\boldsymbol{P}$ and $\boldsymbol{Q}$ were calibrated according to given $m, n, \rho\left(T_{i}, U_{i}\right)$ and $\rho\left(U_{i}, T_{i+1}\right)$. This example illustrates that second order properties by themselves are not sufficient to characterize ruin probabilities; however, it is remarkable that we obtained a deviation of maximum $2 \%$ when varying $m$ and $n$, and in most cases much less. Further improved robustness may be obtained by the inclusion of additional descriptors like higher order (cross) moments in the calibration.

In the following examples, we consider the inter-arrival distribution to be $\operatorname{Er}_{2}(2.5)$ and claim sizes $\mathrm{PH}_{5}(\boldsymbol{\beta}, \boldsymbol{T})$ distributed, where the latter is a maximum likelihood estimation (minimum Kullback-Leibler divergence) to the $\log$-normal $\operatorname{LN}(1,1)$ distribution with density $f(x)=$ $(x \sqrt{2 \pi})^{-1} e^{-(\log (x)-1)^{2} / 2}$. Such a fitting was performed following the algorithm of Asmussen et al. (1996), resulting in the density shown in Figure 6 . The parameters are given by $\boldsymbol{\beta}=(1,0,0,0,0)$ and

$$
T=\left(\begin{array}{ccccc}
-0.778540 & 0.778540 & 0.000000 & 0.000000 & 0.000000 \\
0.000000 & -3.956748 & 3.956748 & 0.000000 & 0.000000 \\
0.000000 & 0.000000 & -3.956739 & 2.019178 & 0.000000 \\
0.000000 & 1.109128 & 0.000000 & -1.578317 & 0.469178 \\
0.644920 & 0.000000 & 0.000000 & 0.000000 & -0.644920
\end{array}\right)
$$

Example 7.3 (Erlangization study of Parisian ruin): Consider a classical Sparre-Andersen process (independent claim sizes and inter-arrival times) with inter-arrival distribution $\operatorname{Er}_{2}(2.5)$ and the claim size distribution $\mathrm{PH}_{5}(\boldsymbol{\beta}, \boldsymbol{T})$. We consider a fixed time clock of $T=25$.

We make a numerical comparison of the probability of Parisian ruin with Erlang clocks $L^{(n)}$, $\psi_{i}^{L^{(n)}}(u)$, and the approximation

$$
\widehat{\psi}_{i}^{T, n}(u)=(n+1) \psi_{i}^{L^{(n+1)}}(u)-n \psi_{i}^{L^{(n)}}(u) \approx \psi_{i}^{T}(u)
$$

suggested by (19). The results are presented in Figure 7 and Table 5. While the convergence of $\psi_{i}^{L^{(n)}}(u)$ as $n \rightarrow \infty$ is slow, the asymptotic approximation $\widehat{\psi}_{i}^{T, n}(u)$ converges quickly and provides a good approximation to $\psi_{i}^{T}(u)$ even for $n \leq 5$. 


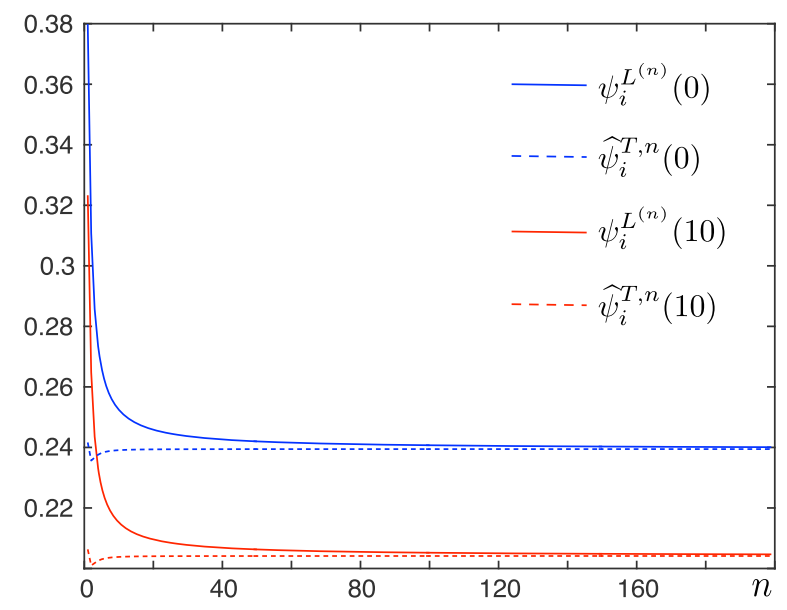

Figure 7. Approximation of $\psi_{i}^{p}(u, T)$ by $\psi_{i}^{p}\left(u, L^{(n)}\right)$ and $\psi_{i}^{\mathrm{app}}(u, T)$ with $T=25$.

Table 5. Approximation of $\psi_{i}^{p}(u, T)$ by $\psi_{i}^{p}\left(u, L^{(n)}\right)$ and $\psi_{i}^{\text {app }}(u, T)$ with $T=25$.

\begin{tabular}{lccccc}
\hline & \multicolumn{2}{c}{$u=0$} & & \multicolumn{2}{c}{$u=10$} \\
\cline { 2 - 3 }$n$ & Erlang & app & & Erlang & app \\
\hline 1 & 0.3799 & 0.2416 & 0.1707 & 0.1090 \\
5 & 0.2663 & 0.2382 & 0.1199 & 0.1073 \\
10 & 0.2525 & 0.2391 & 0.1137 & 0.1077 \\
20 & 0.2459 & 0.2394 & 0.1107 & 0.1078 \\
50 & 0.2420 & 0.2394 & 0.1090 & 0.1078 \\
100 & 0.2407 & 0.2394 & 0.1084 & 0.1078 \\
200 & 0.2401 & 0.2395 & 0.1081 & 0.1078 \\
400 & 0.2398 & 0.2395 & 0.1080 & 0.1078 \\
\hline
\end{tabular}

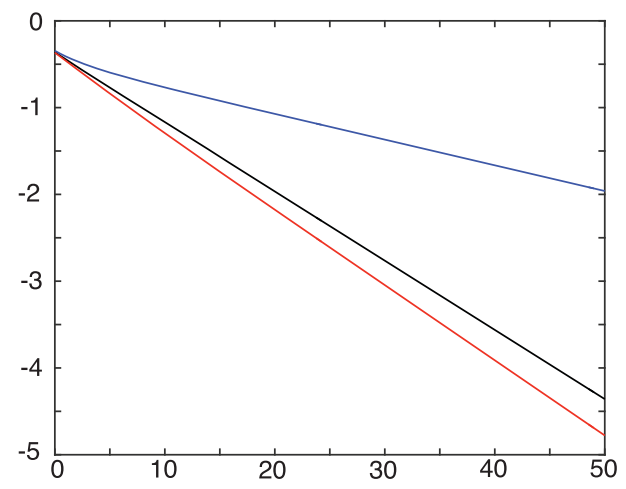

(a)

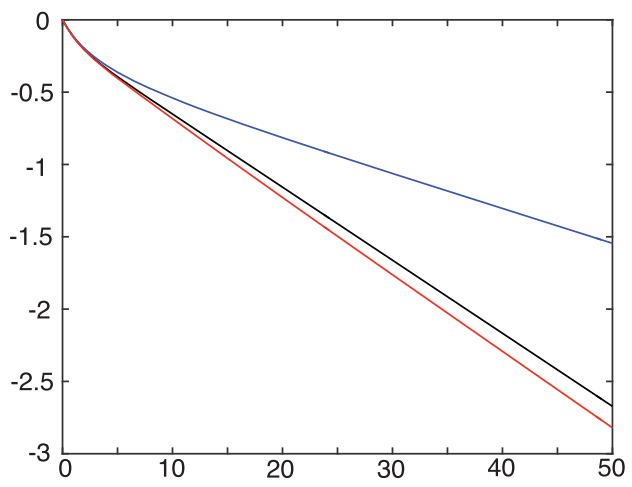

(b)

Figure 8. Log-infinite time-horizon probability of ruin for independent (black), positive-correlated (blue) and negative-correlated (red) claim sizes. (a) Without Brownian noise. (b) With Brownian noise.

Example 7.4 (Correlated claim sizes): We present some numerical results for the probability of infinite time-horizon ruin (Figure 8), Parisian ruin (Figure 9) and cumulative Parisian ruin (Figure 10) for risk models with claims arriving according to a renewal process with $\operatorname{Er}_{2}(2.5)$-distributed interarrival times, and with claim sizes being $\mathrm{PH}_{5}(\boldsymbol{\beta}, \boldsymbol{T})$ distributed, serially correlated but independent 


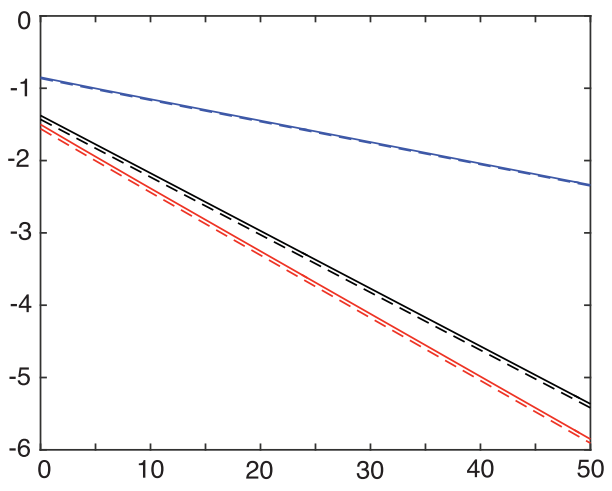

(a)

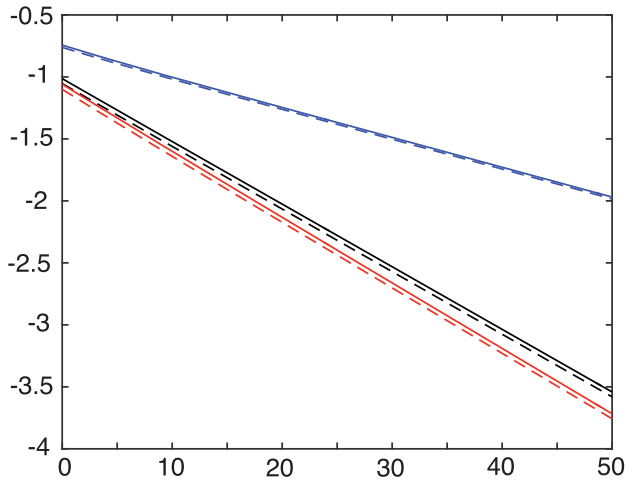

(b)

Figure 9. Log-Parisian probability of ruin for independent (black), positive-correlated (blue) and negative-correlated (red) claim sizes. The dashed lines correspond to asymptotic approximations using (19). (a) Without Brownian noise. (b) With Brownian noise.

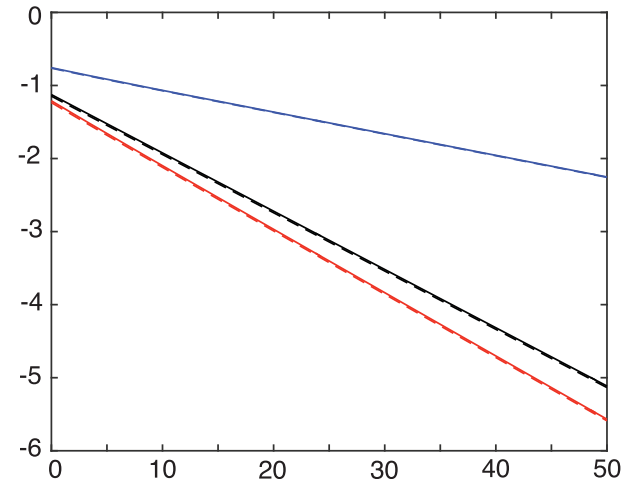

(a)

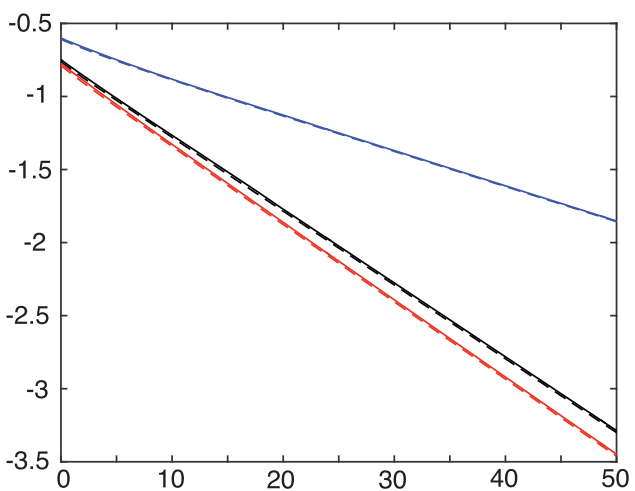

(b)

Figure 10. Log-cumulative Parisian probability of ruin for independent (black), positive-correlated (blue) and negative-correlated (red) claim sizes. The dashed lines correspond to asymptotic approximations using (19). (a) Without Brownian noise. (b) With Brownian noise.

of the inter-arrival times. We consider both cases with and without Brownian noise. The clocks are assumed to be $\operatorname{Er}_{10}(2.5)$ distributed.

In each figure, we compare how these probabilities behave in the case of no correlation (black line), positive correlation of 0.3 (blue line) and negative correlation of -0.3 (red line). The asymptotic approximations to the ruin probability for $T=25$, using formula (32) with $n=10$, are illustrated with dashed lines.

While the presence of a Brownian noise does have an increasing effect on the probability of ruin, which is partly due to the additional possibility of getting ruined between claims and to an overall increased variability of the process, a substantially more important factor seems to be the correlation between claims. Not surprisingly a positive correlation between claim sizes increases the risk of ruin while a negative correlation decreases it.

\section{Disclosure statement}

No potential conflict of interest was reported by the authors. 


\section{Funding}

OP is supported by the CONACYT PhD Scholarship No. 1410763 sponsored by the Mexican Government.

\section{References}

Albrecher, H. \& Boxma, O.J. (2004). A ruin model with dependence between claim sizes and claim intervals. Insurance: Mathematics and Economics 35(2), 245-254.

Albrecher, H., Boxma, O.J. \& Ivanovs, J. (2014). On simple ruin expressions in dependent Sparre Andersen risk models. Journal of Applied Probability 51(01), 293-296.

Albrecher, H. \& Ivanovs, J. (2017). Strikingly simple identities relating exit problems for Lévy processes under continuous and Poisson observations. Stochastic Processes and their Applications 127(2), 643-656.

Asmussen, S. (1995). Stationary distributions for fluid flow models with or without Brownian noise. Stochastic Models 11(1), 21-49.

Asmussen, S. (1995). Stationary distributions via first passage times. In Advances in queueing: theory, methods, and open problems. Ed: J. H. Dshalalow. Boca Raton: CRC Press. p. 79-102.

Asmussen, S. (2003). Applied Probability and Queues (Stochastic Modelling and Applied Probability). New York: Springer. Asmussen, S., Avram, F. \& Usabel, M. (2002). Erlangian approximations for finite-horizon ruin probabilities. ASTIN Bulletin: The Journal of the IAA 32(2), 267-281.

Asmussen, S., Nerman, O. \& Olsson, M. (1996). Fitting phase-type distributions via the EM algorithm. Scandinavian Journal of Statistics 23(4), 419-441.

Assaf, D., Langberg, N.A., Savits, T.H. \& Shaked, M. (1984). Multivariate phase-type distributions. Operations Research 32(3), 688-702.

Avram, F., Badescu, A.L., Pistorius, M.R. \& Rabehasaina, L. (2016). On a class of dependent Sparre Andersen risk models and a bailout application. Insurance: Mathematics and Economics 71, 27-39.

Badescu, A., Breuer, L., da Silva Soares, A., Latouche, G., Remiche, M.-A. \& Stanford, D. (2005). Risk processes analyzed as fluid queues. Scandinavian Actuarial Journal 2005(2), 127-141.

Badescu, A.L., Cheung, E.C.K. \& Landriault, D. (2009). Dependent risk models with bivariate phase-type distributions. Journal of Applied Probability 46(01), 113-131.

Badescu, A.L. \& Landriault, D. (2009). Applications of fluid flow matrix analytic methods in ruin theory: a review. Revista de la Real Academia de Ciencias Exactas, Físicas y Naturales. Serie A.Matemáticas 103(2), 353-372.

Badila, E.S., Boxma, O.J. \& Resing, J.A.C. (2014). Queues and risk processes with dependencies. Stochastic Models 30(3), 390-419.

Badila, E.S., Boxma, O.J. \& Resing, J.A.C. (2015). Two parallel insurance lines with simultaneous arrivals and risks correlated with inter-arrival times. Insurance: Mathematics and Economics 61, 48-61.

Baker, R. (2008). An order-statistics-based method for constructing multivariate distributions with fixed marginals. Journal of Multivariate Analysis 99(10), 2312-2327.

Baurdoux, E.J., Pardo, J.C., Perez, J.L. \& Renaud, J.-F. (2016). Gerber-Shiu distribution at Parisian ruin for Lévy insurance risk processes. Journal of Applied probability 53, 572-584.

Bean, N.G., O’Reilly, M.M., Taylor, P.G. (2005). Algorithms for return probabilities for stochastic fluid flows. Stochastic Models 21(1), 149-184.

Bean, N.G., O’Reilly, M. \& Taylor, P.G. (2005). Hitting probabilities and hitting times for stochastic fluid flows. Stochastic Processes and their Applications 115(9), 1530-1556.

Bladt, M. (2005). A review on phase-type distributions and their use in risk theory. Astin Bulletin 35(1), $145-161$.

Bladt, M. \& Nielsen, B.F. (2010). Multivariate matrix-exponential distributions. Stochastic Models 26(1), 1-26.

Bladt, M. \& Nielsen, B.F. (2010). On the construction of bivariate exponential distributions with an arbitrary correlation coefficient. Stochastic Models 26(2), 295-308.

Bladt, M. \& Nielsen, B.F. (2017). Matrix-exponential distributions in applied Probability. Probability Theory and Stochastic Modelling. New York: Springer Verlag.

Borst, S.C., Boxma, O.J. \& Combé, M.B. (1992). Collection of customers: a correlated M/G/1 queue. In ACM SIGMETRICS Performance Evaluation Review. New York: ACM. Volume 20, P. 47-59.

Boudreault, M., Cossette, H., Landriault, D. \& Marceau, E. (2006). On a risk model with dependence between interclaim arrivals and claim sizes. Scandinavian Actuarial Journal 2006(5), 265-285.

Conolly, B.W. (1968). The waiting time process for a certain correlated queue. Operations Research 16(5), 1006-1015.

Czarna, I., Li, Y., Palmowski, Z. \& Zhao, C. (2017). The joint distribution of the parisian ruin time and the number of claims until parisian ruin in the classical risk model. Journal of Computational and Applied Mathematics 313, 499-514.

Czarna, I. \& Palmowski, Z. (2011). Ruin probability with Parisian delay for a spectrally negative Lévy risk process. Journal of Applied Probability 48, 984-1002.

Dassios, A. \& Wu, S. (2008). Parisian ruin with exponential claims. Department of Statistics, London School of Economics and Political Science, London (Unpublished). 
Dou, X., Kuriki, S. \& Lin, G.D. (2013). Dependence structures and asymptotic properties of Baker's distributions with fixed marginals. Journal of Statistical Planning and Inference 143(8), 1343-1354.

Guérin, H. \& Renaud, J.-F. (2017). On the distribution of cumulative parisian ruin. Insurance: Mathematics and Economics 73, 116-123.

Kulkarni, V.G. (1989). A new class of multivariate phase type distributions. Operations research 37(1), 151-158.

Landriault, D., Renaud, J.-F. \& Zhou, X. (2014). An insurance risk model with Parisian implementation delays. Methodology and Computing in Applied Probability 16(3), 583-607.

Lin, G.D. \& Huang, J.S. (2010). A note on the maximum correlation for Bakers bivariate distributions with fixed marginals. Journal of Multivariate Analysis 101(9), 2227-2233.

Loeffen, R., Palmowski, Z. \& Surya, B.A. (In press). Discounted penalty function at parisian ruin for Lévy insurance risk process. Insurance: Mathematics and Economics

Loeffen, R., Czarna, I. \& Palmowski, Z. (2013). Parisian ruin probability for spectrally negative Lévy processes. Bernoulli 19(2), 599-609.

Müller, A., (2000). On the waiting times in queues with dependency between interarrival and service times. Operations Research Letters 26(1), 43-47.

Ramaswami, V. (2006). Passage times in fluid models with application to risk processes. Methodology and Computing in Applied Probability 8(4), 497-515. 